\title{
SYSTEMATIC REVISION OF THE GENUS CEPHALOCYCLUS WITH DESCRIPTION OF TEN NEW SPECIES FROM MEXICO AND COSTA RICA(SCARABAEOIDEA: APHODIIDAE)
}

\author{
Marco Dellacasa ${ }^{1}$, Giovanni Dellacasa ${ }^{2}$ \& Robert D. Gordon ${ }^{3}$ \\ ${ }^{1}$ Museo di Storia naturale e del Territorio, Università di Pisa. Via Roma, 79. 56011 Calci (Pisa)-ITALY \\ E-mail: dellacasa@museo.unipi.it \\ ${ }^{2}$ C. P. 921,16121 Genova-ITALY \\ ${ }^{3}$ Northern Plains Entomology, P.O. Box 65, Willow City, ND 58384 - U.S.A. \\ E-mail:rdgordon@utma.com
}

\begin{abstract}
RESUMEN
El género Cephalocyclus Dellacasa M., Gordon \& Dellacasa G. es revisado por primera vez. Se describen diez nuevas especies: Cephalocyclus bordati, C. carmenae, C. cartagoensis, C. costaricensis, $C$. halffteri, C. howdenorum, C. lagoi, C. ordonezi, C. potosinus, and C. rockefelleri. Aphodius luteolus Horn, 1887 es transferido al género Cephalocyclus; Aphodius pugil Balthasar, 1946 resulta ser una sinonimia menor de Cephalocyclus durangoensis (Bates, 1887) y Aphodius transversus Robinson, 1940 resultó una sinonimia menor de Cephalocyclus hogei (Bates,1887). Se presenta una clave para las 21 especies conocidas de Cephalocyclus así como las descripciones e ilustraciones de todos los taxones.

Palabras Clave: Aphodiidae, Cephalocyclus, nuevas especies, nueva combinación, nuevos sinonimias, taxonomy, distribución, México, Costa Rica.
\end{abstract}

\begin{abstract}
The genus Cephalocyclus Dellacasa M., Gordon \& Dellacasa G. is revised for the first time. Ten new species are described: Cephalocyclus bordati, C. carmenae, C. cartagoensis, C. costaricensis, C. halffteri, C. howdenorum, C. lagoi, C. ordonezi, C. potosinus, and C. rockefelleri. Aphodius luteolus Horn, 1887 is transferred into genus Cephalocyclus; Aphodius pugil Balthasar, 1946 resulted a junior synonym of Cephalocyclus durangoensis (Bates, 1887) and Aphodius transversus Robinson, 1940 resulted a junior synonym of Cephalocyclus hogei (Bates, 1887). A key to the presently known 21 species of Cephalocyclus as well as descriptions and illustrations of all taxa are supplied.

Key Words: Aphodiidae, Cephalocyclus, new species, new combination, new synonymies, taxonomy, distribution, Mexico, Costa Rica.
\end{abstract}

\section{INTRODUCTION}

The genus Cephalocyclus Dellacasa M., Gordon and Dellacasa G. contains 21 species that occur in Costa Rica, Guatemala, Mexico, and the United States. It is a 
relatively large genus among the Mexican and Central American genera of Aphodiini, and is here revised for the first time. Cephalocyclus species apparently feed on surface dung deposits as indicated by label data and collecting notes and many are attracted to various forms of light at night, but no research has been published concerning the biological details.

In previous papers we discussed the species of Aphodius described by Harold (Dellacasa M. \& alii, 1998), Schmidt A. (Dellacasa M. \& alii, 2000) and Bates (Dellacasa M. \& alii, 2002), that belong to the genus Cephalocyclus, as well as $C$. stebnickae Deloya \& Ibáñez-Bernal, 2000.

Examination of type material of three additional taxa indicates that Aphodius luteolus LeConte, Aphodius pugil Balthasar, and Aphodius transversus Robinson are also members of Cephalocyclus, and are treated as follows:

- Aphodius luteolus Horn (1887) is a valid species;

- Aphodius pugil Balthasar (1946) is a junior synonym of Cephalocyclus durangoensis (Bates, 1887);

- Aphodius transversus Robinson (1940) is a junior synonym of Cephalocyclus hogei (Bates, 1887).

Examination of Aphodiinae material preserved in several public or private collections revealed several specimens that are here described as new species. We supply a key to the presently known species of Cephalocyclus as well as descriptions and illustrations of all taxa.

Repositories: Under material examined we include only specimens not recorded in previous publications. These specimens are preserved in the following collections:

AMNHNY: American Museum of Natural History, New York (USA)

BACB: Bellman A. Collection, Bremen (Germany)

CNCO: Canadian National Collection, Ottawa (Canada)

CSCAG: California State Collection of Arthropods, California Department of Food and Agriculture, Sacramento (USA)

DBUMU: Department of Biology, University of Mississippi, University (USA)

DACW: Dostal A. Collection, Wien (Austria)

DCG: Dellacasa Collection, Genoa (Italy)

EMEB: Essig Museum of Entomology, University of California, Berkeley (USA)

HAHCO: Howden H. \& A. Collection, Ottawa (Canada)

IEX: Instituto de Ecología A.C., Xalapa (Veracruz, Mexico)

LSAMBR: Louisiana State Arthropod Museum, Department of Entomology,

Louisiana State University, Baton Rouge (USA)

MSNTC: Museo di Storia Naturale e del Territorio, Università di Pisa, Calci (Pisa, Italy) 
RTC: Tornbow R. Collection, Gainesville (USA)

UNAM: Museo de Zoología, FES Zaragoza, U.N.A.M., Iztapalapa, D.F. (Mexico)

USNMW: United States National Museum of Natural History, Smithsonian Institution, Washington (USA)

\section{GENUS CEPHALOCYCLUS DELLACASA M., GORDON \& DELLACASAG., 1998}

Aphodius (Platyderus) Schmidt A., 1913: 123 (nec Platyderus J. F. Stephens, 1828, Col. Carabidae) (partim).

Aphodius (Platyderides) Schmidt A., 1916: 99 (nomen novum) (partim).

Cephalocyclus Dellacasa M. \& alii, 1998: 141; Deloya \& Ibáñez-Bernal, 2000: 322; Dellacasa G. \& Alii, 2001: 114; Dellacasa M. \& alii, 2002: 213.

Type species: Aphodius fuliginosus Harold, 1863 (original designation).

Diagnosis: small or medium size species (length 3.5-9.0 mm), moderately convex; dull, rarely shiny; elytra glabrous or more or less diffusedly pubescent. Piceous either pale or dark testaceous; elytra paler. Head with epistoma nearly flat, punctured; clypeus nearly semicircular, faintly truncate or feebly sinuate at middle, widely rounded at sides, quite thinly bordered, edge thickened at middle, elongately bristled at sides; genae strongly developed, abruptly angulate, not deflexed, bristled, protruding much more than eyes; latter medium size; frontal suture finely impressed, not at all tuberculate. Pronotum widely transverse, explanate at sides; lateral margins thinly bordered, elongately bristled; hind angles quite rounded, feebly depressed; base bisinuate, not bordered. Scutellum small, triangular. Elytra moderately convex, oval-elongate, not denticulate at shoulder, more or less widened posteriorly and more or less strongly callose on preapical declivity, more or less diffusedly pubescent or nearly glabrous, usually rather dull; striae generally fine and feebly or not at all crenulate; interstices flat, rarely convex, more or less distinctly punctured; epipleura strong, elongately bristled at least at two basal thirds. Fore tibiae distally tridentate and proximally serrulate at outer margin; upper side smooth. Hind tibiae with strong transverse carinae on outer face, apically fimbriate with spinules very elongate and irregularly unequal. Hind tibiae superior apical spur as long as or longer than first tarsomere; latter as long as following three combined. Claws strong. Pygidium sparsely and granulosely punctured, punctures shortly and straightly setigerous; its apical margin subcrenulate at middle and therein with dense, fine and elongate cilia. Sexual dimorphism variable from species to species; in males, e. g., head wider and more flat; pronotum relatively more transverse; protibiae, modified or not, with apical spur usually short, stout and abruptly downward curved; middle tibiae inferior apical spur sometimes aberrant; middle tarsi normally shaped or strongly modified. Aedeagus with paramera rather short, curved and acuminate, or more or less rounded and membranous apically. Epipharynx transverse, strongly rounded at sides; anterior margin nearly straight; epitorma conical, at each side with a longitudinal row of stout spines; corypha slightly protruding beyond front margin, usually with apical clump of stout and elongate spiculae; pedia densely pubescent with several short, stout, scattered chaetae; chaetopariae moderately dense and moderately elongate.

Distribution: Southern U.S.A., Mexico, Guatemala, Costa Rica. 
Dellacasa et al.: Revision of genus Cephalocyclus and ten new species

Key to species

1 Clypeal lateral margins not ciliate; pronotum coarsely and rather densely punctured; elytral interstices slightly convex, microreticulate so few shiny, finely distinctly and rather densely punctured. Piceous; clypeal margin and pronotal sides vaguely brownish. Length 7.0-8.5 mm. Mexico (Chiapas) ................................................ stebnickae

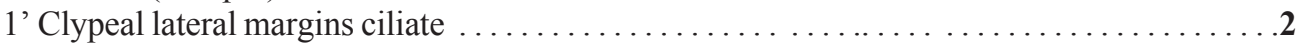

2 Lateral margins of pronotum glabrous ................................. 3

2' Lateral margins of pronotum more or less elongately ciliate or, at least, ciliate toward front angles............................

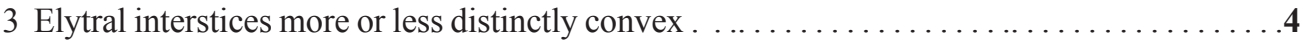

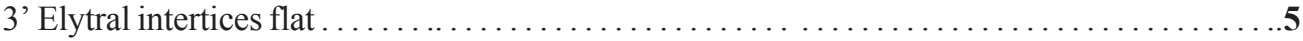

4 Elytra distinctly pubescent on preapical declivity; elytral interstices shiny, coarsely densely punctured. Pale brownish-red; pronotal disc and elytral suture darker. Length 7.0-8.0 mm. Costa Rica (Cartago) ..............................................

4'Elytra almost glabrous, only with few, extremely short, pale hairs irregularly sparse on preapical declivity; elytral interstices microreticulate, evenly distinctly punctured. Head and pronotum blackish; elytra piceous. Length 3.5-4.5 mm. Mexico (Jalisco, Edo. de México, Puebla) ......

puncticeps

5 Epipleural carina subdenticulate at shoulder. Head and pronotum piceous; clypeal margins, pronotal sides and elytra reddish-brown. Length 5.5-6.0 mm. Mexico (Edo. de México, Puebla)

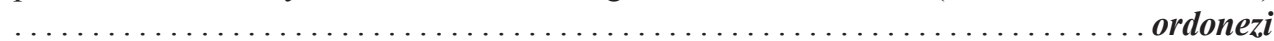

5 ' Epipleural carina not at all denticulate at shoulder ....................... 6

6 Elytral interstices smooth, thus rather shiny, almost imperceptibly punctured, glabrous. Brownish-red; pronotal disc and elytral suture darker. Length 6.0-7.0 mm. Costa Rica (Alejuela, Cartago, San José) . . . . . . . . . . . . . . . . . . . . . . . . . . costaricensis

6' Elytral interstices microreticulate, thus strongly dull. . . . . . . . . . . . . . . . 7

7 Elytral interstices shortly and sparsely pubescent on preapical declivity; pronotum without median longitudinal groove; apical spur of fore tibiae, in males, stout, abruptly downward and outward bent. Piceous; clypeal margins, pronotal sides and elytral suture paler. Length 6.0-7.0 mm. Mexico (Guerrero, Edo. de México) . ........................ halffteri

7' Elytral interstices glabrous; pronotum with feeble median longitudinal groove; apical spur of fore tibiae, in males, abruptly downward and inward bent (fig. 61). Piceous; clypeal margins and pronotal sides paler. Length 5.5-7.0 mm. Mexico (Coahuila, Nuevo León) . . . . carmenae

8 Lateral margins of pronotum elongately ciliate toward front angles only; first mesotarsomere, in males, with elongate infero-apical seta as long as following three tarsomeres combined (fig. 65). Piceous; pronotal sides and underside testaceous. Length 5.0-6.0 mm. Guatemala, Mexico (Distrito Federal, Durango, Guerrero, Hidalgo, Jalisco, Edo. de México, Michoacán, Morelos, Nayarit, Oaxaca, Puebla, Querétaro, Sinaloa) .................. mexicanus

8' Lateral margins of pronotum entirely very shortly bristled or elongately ciliate . . . . . . . 9

9 Lateral margins of pronotum very shortly bristled (fig. 46); shoulder densely elongately pubescent, pubescence diffused on basal elytral margins till near scutellum. Dark brown; clypeal margins, pronotal sides and elytral apex vaguely reddish. Length $4.5-5.0 \mathrm{~mm}$. Mexico

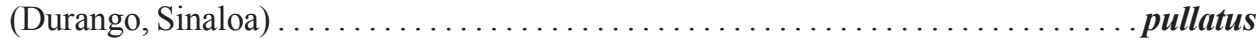


9' Lateral margins of pronotum elongately ciliate $\ldots \ldots \ldots \ldots \ldots \ldots \ldots \ldots \ldots \ldots \ldots$

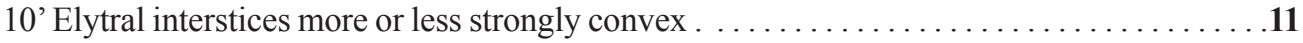



$11^{\prime}$ Elytral interstices sparsely shortly pubescent on preapical declivity $\ldots \ldots \ldots \ldots \ldots \ldots .12$

$11^{\prime}$ Elytral intertices glabrous . . . . . . . . . . . . . . . . . . . . . . . . . . . . 13

12 Upperside faintly microreticulate thus shiny; elytral interstices finely, sparsely but distinctly punctured. Yellowish-brown; pronotal disc and elytral suture darker. Length 6.0-7.0 mm. Mexico (Coahuila, Durango, Michoacán, Nuevo León, Oaxaca).............. durangoensis

12' Upperside more or less strongly microreticulate thus rather dull; elytral interstices almost imperceptibly punctured. Piceous; elytra dark brownish. Length 4.0-6.0 mm. Guatemala, Mexico (Coahuila, Distrito Federal, Durango, Hidalgo, Edo. de México, Michoacán, Morelos, Oaxaca, Puebla, Querétaro, Veracruz), U.S.A. (Arizona)? . . . . . . . . . . . . . . . . . . hogei

13 Elytral interstices superficially microreticulate, at least near striae, thus few shiny $\ldots \ldots \ldots 14$

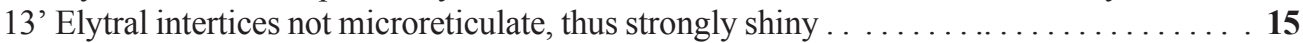

14 Pronotum distinctly microreticulate throughout thus almost dull, irregularly relatively more densely and coarsely punctured mainly on sides and toward hind angles; elytra feebly widened posteriorly. Dark brownish. Length 5.0-5.5 mm. Mexico (Coahuila, Nuevo León, Querétaro, San Luis Potosí) $\ldots \ldots \ldots \ldots \ldots \ldots \ldots \ldots \ldots \ldots \ldots \ldots \ldots \ldots \ldots$ lagoi

14' Pronotum smooth on disc, microreticulate at sides, finely sparsely punctured; elytra subparallel sided. Dark brownish; pronotal sides and elytra testaceous. Length 5.0-6.0 mm. Mexico (Chihuahua, Durango) ......................................... bordati

15 Size relatively larger (length 7.0-8.0 mm); inferior apical spur of middle tibiae, in males, spatulate and outwardly curved, nearly as long as first mesotarsomere (fig. 67). Dark brownish. Mexico (Distrito Federal, Oaxaca, Puebla) ................... luridiventris

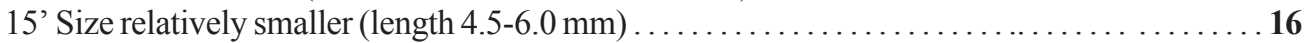

16 Entirely reddish-brown; inferior apical spur of middle tibiae, in males, spatulate and outwardly curved; first mesotarsomere cylindrically plumped and distinctly longitudinally grooved inwardly (fig. 66). Length $4.5-6.0 \mathrm{~mm}$. Mexico (Chihuahua) ................. rockefelleri

16 ' Head, pronotum and juxtasutural interstices brownish, elytra yellowish; inferior apical spur of middle tibiae and first mesotarsomere normally shaped in both sexes. Length 5.0-6.0 mm. U.S.A. (Arizona, New Mexico, Texas, Utah) . . . . . . . . . . . . . . . . . . . . . . . luteolus

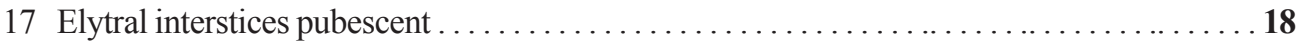

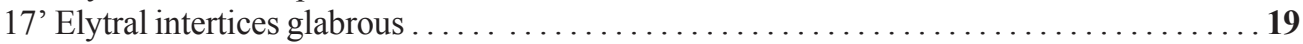

18 Elytral pubescence more or less widely diffused laterally and on preapical declivity; elytra oblong; mesotarsomeres II-IV, in males, distinctly plumped (fig. 64). Dark piceous; sometimes elytra pale brownish. Length 5.0-7.0 mm. Guatemala, Mexico (Chihuahua, Distrito Federal, Durango, Guanajuato, Hidalgo, Jalisco, Edo. de México, Michoacán, Morelos, Nayarit, Oaxaca, Puebla, Sinaloa, Tlaxcala, Zacatecas) ........................fuliginosus

18' Elytral pubescence on preapical declivity only; elytra distinctly broadened posteriorly; mesotarsomeres normally shaped in both sexes. Piceous; clypeal margins, pronotal sides and elytra brownish. Length 6.0-8.0 mm. Mexico (Chihuahua, Distrito Federal, Durango, Hidalgo, Jalisco, Edo. de México, Michoacán) ........................ gravidus

19 Size relatively smaller (length 5.0-6.0 mm); epipleural carina distinct at shoulder. Head and pronotum piceous, elytra reddish-brown. Mexico (Coahuila, Nuevo León) .......... potosinus 
19' Size relatively larger (length 7.0-9.0 mm); epipleural carina not distinct at shoulder .. . . . . 20

20 Elytral interstices distinctly microreticulate thus almost dull; inferior apical spur of middle tibiae, in males, spatulate, outwardly curved and as long as the half of the first mesotarsomere (fig. 68). Dark reddish-brown. Length 8.0-9.0 mm. Mexico (Hidalgo, Edo. de México, Oaxaca,

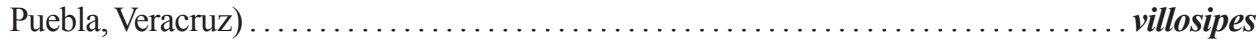

20' Elytral intertices not microreticulate thus very shiny; inferior apical spur of middle tibiae normally shaped in both sexes. Dark piceous; clypeal margins and pronotal sides somewhat paler, elytra dark brownish-yellow. Length 7.0-8.0 mm. Mexico (Nuevo León) ..... howdenorum

\section{CEPHALOCYCLUS BORDATI SP. NOV.}

(Figs. 1-3)

Type locality: San José Babicora, Chihuahua, Mexico.

Type repository: American Museum of Natural History. New York.

Description: length 5.0-6.0 mm, elongate, convex, shiny, glabrous. Head and pronotum dark brownish, sides of the latter and elytra testaceous; legs brown-testaceous with paler tarsi; antennal club yellowish. Head with epistoma feebly convex on disc, finely subregularly sparsely punctured, punctation somewhat denser and coarser distally; clypeus faintly sinuate at middle, rounded at sides, almost thickly bordered, the edge upturned; genae subauriculate, obtusely angulate, elongately ciliate, distinctly protruding more than eyes; frontal suture finely impressed, nearly faint at middle; front relatively somewhat densely punctured. Pronotum moderately transverse, doubly punctured, large punctures, two or three times larger than small ones, arranged laterally, almost regularly convex on disc, widely flattened at sides mainly toward front and hind angles, and thickly bordered, the edge elongately almost sparsely ciliate; hind angles obtusely truncate; base bisinuate. Scutellum depressed, microreticulate and superficially punctured at middle toward the base. Elytra elongate, subparallel-sided; striae distinctly impressed, superficially punctured, subcrenulate; interstices feebly convex, finely microreticulate laterally, smooth at middle, very sparsely and finely punctured. Hind tibiae superior apical spur as long as first tarsomere; latter as long as following three combined. Male: head relatively wider, more flat and more shiny; pronotum not narrowed frontward and more sparsely finely punctured; fore tibiae more elongate, slender, somewhat widened anteriorly, third outer tooth very feeble and apical spur inwardly curved; aedeagus fig. 3. Female: head relatively narrower, more convex and less shiny; pronotum narrowed frontward and more densely coarsely punctured; fore tibiae stouter, not widened anteriorly, third outer tooth large and apical spur straight.

Collecting notes: none.

Distribution: Mexico (Chihuahua, Durango).

Derivatio nominis: named in honour of Patrice Bordat, specialist on Afrotropical Aphodiinae in Saint-Cirq (France).

Type material: Mexico - Chihuahua: San José Babicora, 5.VII.1947, leg. Cazier, D. Rockefeller Exp., 5 exx. (paratypes, AMNHNY); idem, leg. Gertsch, D. Rockefeller Exp., 8 exx. (paratypes, AMNHNY); idem, leg. Michener, D. Rockefeller Exp., 28 exx. (holotype male, allotype female, paratypes, AMNHNY, DCG); idem, leg. Schramel, D. Rockefeller Exp., 3 exx. (paratypes, AMNHNY); Durango: Las Puentes, 7500', 24.VII.1947, leg. Cazier, D. Rockefeller Exp., 2 exx. (paratypes, AMNHNY); idem, leg. Gertsch, D. Rockefeller Exp., 6 exx. (paratypes, AMNHNY, DCG). 


\section{CEPHALOCYCLUS CARMENAE SP. NOV.}

\section{(Figs. 61-63)}

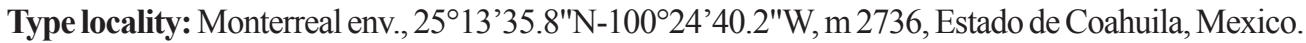
Type repository: Dellacasa Collection, Genoa.

Description: length 5.5-7.0 mm, oblong, convex; dull, glabrous. Piceous; clypeal margins and pronotum sides paler; legs reddish-brown; antennal club rufo-testaceous. Head with epistoma feebly convex on disc, finely microreticulate, distally confusedly rugose, on disc finely, sparsely and regularly punctured; clypeus subtruncate anteriorly, widely rounded laterally, thickly bordered, the edge feebly upturned and sparsely, rather shortely ciliate laterally; genae subauriculate, obtusely rounded, sparsely elongately ciliate, distinctly protruding more than eyes; frontal suture distinctly impressed; front distinctly regularly punctured. Pronotum moderately transverse, strongly convex with feeble median longitudinal groove shortened anteriorly, dually subregularly punctured, large punctures three times larger than small punctures; sides arcuate, thickly bordered, edge glabrous; hind angles widely rounded; base bisinuate, not bordered. Scutellum irregularly punctured on basal half. Elytra elongate, feebly widened posteriorly, strongly convex, superficially striate; striae faintly punctured, not crenulate; interstices flat, distinctly microreticulate, very sparsely and finely punctured. Hind tibiae superior apical spur somewhat shorter than first tarsomere; latter almost as long as the following three combined. Male: head relatively wider, less convex; pronotum more transverse; elytra less widened posteriorly; fore tibiae apical spur abruptly downward and inward bent; aedeagus fig. 63. Female: head relatively narrower, more convex; pronotum narrower frontward; elytra more widened posteriorly; fore tibiae apical spur moderately downward and outward bent.

Collecting notes: found in horse dung.

Distribution: Mexico (Coahuila, Nuevo León).

Derivatio nominis: named in honour of Dr. Carmen Fresi, fiancée of M. Dellacasa.

Type material: Mexıco - Coahuila: Paraíso Escondido, 25¹3’29.0"N-100²3’29.8"W, m 2476, 5.VII.2006, leg. Dellacasa M., Fresi C. \& Martínez I., 44 exx. (paratypes, DCG, MSNTC); Nuevo León: Monterreal env., $2^{\circ} 13^{\prime} 35.8^{\prime \prime N}-100^{\circ} 24^{\prime} 40.2^{\prime \prime} \mathrm{W}, \mathrm{m} 2736,3$.VII.2006, leg. Dellacasa M., Fresi C. \& Martínez I., horse dung, 19 exx. (holotype, allotype, paratypes, DCG).

\section{CEPHALOCYCLUS CARTAGOENSIS SP. NOV.}

(Figs. 4-6)

Type locality: Cartago, Costa Rica.

Type repository: Canadian National Collection. Ottawa.

Description: length 7.0-8.0 mm, elongate, convex, moderately shiny, elytral preapical declivity distinctly pubescent. Pale brownish; pronotal disc and elytral suture dark brown; antennal club dark testaceous. Head with epistoma feebly gibbous at centre, irregularly punctured, punctation distally denser and rather coarse, on disc fine and very sparse; clypeus anteriorly subtruncate, widely rounded at sides, rather thickly bordered, the edge feebly upturned and distinctly bristled laterally; genae angulate, posteriorly $09^{\circ} 34^{\prime} \mathrm{N}-83^{\circ} 41^{\prime}$ W, VIII.1976, leg. Hespenheide H. A., 1 ex. (holotype, male, HAHCO); San José env., m 1000-1200, VII.1962, 8 exx. (paratypes, DCG); San José 
Dellacasa et al.: Revision of genus Cephalocyclus and ten new species
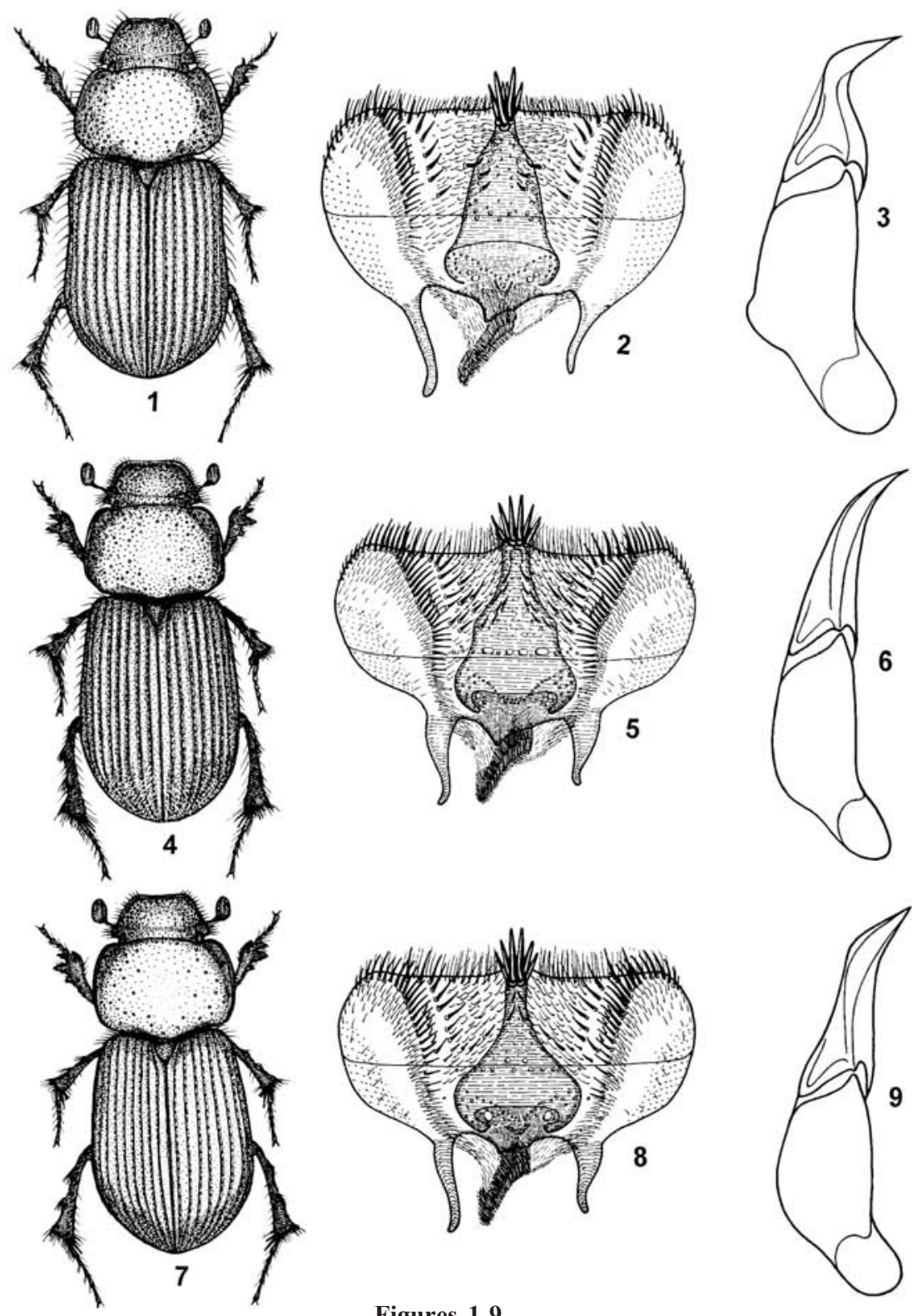

Figures 1-9

1-3.- Cephalocyclus bordati: 1.- habitus of male (length $5.0 \mathrm{~mm}$; San José Babicora, Edo. Chihuahua, Mexico); 2.- epipharynx; 3.- aedeagus (lateral view); 4-6.- Cephalocyclus cartagoensis: 4.- habitus of male; (length 8.0 mm; Paraíso, Cartago Province, Costa Rica); 5.- epipharynx; 6.- aedeagus (lateral view); 7-9.Cephalocyclus costaricensis: 7.- habitus of male; (length $7.0 \mathrm{~mm}$; Villa Mills (La Georgina), San José Province, Costa Rica); 8.- epipharynx; 9.- aedeagus (lateral view). 
Prov., Tres de Junio, Interamericana S., 2700 m, 4.V.2001, leg. Hielkema M.A. (cow dung), 3 exx. (paratypes, BACB, DCG); San José prov., Villa Mills, $09^{\circ} 34^{\prime} \mathrm{N}-83^{\circ} 41^{\prime}$ W, 6.V.1968, leg. Hunt J. H., 1 ex. (paratype, HAHCO); San José-Cartago prov., Cerro de la Muerte, 10000', 2.VIII.1966, leg. Peck S., 2 exx. (paratypes, HAHCO); idem, Mirador de Quetzales, $9^{\circ} 39^{\prime} \mathrm{N}-83^{\circ} 51^{\prime} \mathrm{W}, \mathrm{m}$ 2700, 13-18.V.2006, leg. Holzer E., 18 exx. (paratypes, BACB, DCG); S. José/Cart., km 55, Int. Amer. Hwy., El Empalme 3 km S., 0942’30"N-8357’W, m 2350, 8.VI.1997, leg. Anderson R., oak forest litter 97-008D, 1 ex. (paratype, CNCO); S. José/Cart., km 68, Int. Amer. Hwy., nr. Tres de Junio, 09³9’46"N-8350’59"W, m 2560, 18.VI.1998, leg. Anderson R., wet cloud for. litter 98-101C, 1 ex. (paratype, CNCO); S. José/ Cart., km 72, Int. Amer. Hwy., Ojo de Agua 3 km W., 09³7’30"N-8350’30"W, m 2950, 7.VI.1997, leg. Anderson R., oak for. litter 97-005A, 1 ex. (paratype, CNCO).

\section{CEPHALOCYCLUS COSTARICENSIS SP. NOV.}

(figs 7-9)

Type locality: La Georgina, 1 mi. N W Villa Mills, San José prov., Costa Rica.

Type repository: Howden Collection, Ottawa.

Description: length 6.0-7.0 mm, oblong, moderately convex, very shiny, glabrous. Brownreddish; pronotal disc and elytral suture darker; antennal club testaceous. Head with epistoma feebly gibbous at centre, irregularly punctured, punctation denser and coarser distally, very superficial and sparse on disc; clypeus subtruncate anteriorly, widely rounded at sides, distinctly bordered, the edge rather elongately bristled laterally; genae angulate, elongately sparsely ciliate, protruding more than eyes; frontal suture finely impressed laterally only; front very finely and sparsely punctured. Pronotum transverse, moderately convex, dually sparsely irregularly punctured; large punctures, two or three times larger than small ones, somewhat denser and coarser on sides, missing on disc; small punctures some more distinct on sides, very fine, superficial and sparse on disc; sides feebly arcuate, thickly bordered, the edge glabrous; hind angles obtusely rounded; base distinctly bisinuate. Scutellum rather wide, irregularly sparsely punctured. Elytra oval-elongate, feebly widened posteriorly, striae rather deep, distinctly punctured, feebly crenulate; interstices flat, almost imperceptibly sparsely punctured. Hind tibiae superior apical spur as long as first tarsomere; latter somewhat shorter than following three combined. Male: head relatively wider; pronotum more transverse and less convex; inferior apical spur of middle tibiae truncate apically; aedeagus fig. 9. Female: head relatively narrower; pronotum less transverse and more convex; inferior apical spur of middle tibiae regularly acuminate.

Collecting notes: none.

Distribution: Costa Rica (Alejuela, Cartago, San José).

Etimology: the species is named after its type locality.

Type material: Costa Rica: Alejuela Prov., Poas, $10^{\circ} 09.380^{\prime} \mathrm{N}-084^{\circ} 14.010^{\prime} \mathrm{W}, \mathrm{m} 2225,16 . \mathrm{VI} .2004$, leg. Dostal \& Uhler, 10 exx. (paratypes, DACW, DCG); San José prov., La Georgina, 1 mi. NW Villa Mills, $09^{\circ} 34^{\prime}$ N-834ㄴ'W, VIII.1976, leg. Hespenheide H. A., 1 ex. (holotype, male, HAHCO); San José env., m 1000-1200, VII.1962, 8 exx. (paratypes, DCG); San José Prov., Tres de Junio, Interamericana S., 2700 m, 4.V.2001, leg. Hielkema M.A. (cow dung), 3 exx. (paratypes, ВАСB, DCG); San José prov., Villa Mills, $09^{\circ} 34^{\prime}$ N-834ㄴ'W, 6.V.1968, leg. Hunt J. H., 1 ex. (paratype, 
нансо); San José-Cartago prov., Cerro de la Muerte, 10000', 2.VIII.1966, leg. Peck S., 2 exx. (paratypes, HAHCO); idem, Mirador de Quetzales, $9^{\circ} 39^{\prime} \mathrm{N}-83^{\circ} 51^{\prime} \mathrm{W}, \mathrm{m}$ 2700, 13-18.V.2006, leg. Holzer E., 18 exx. (paratypes, вACB, DCG); S. José/Cart., km 55, Int. Amer. Hwy., El Empalme 3 km S., 09² $2^{\prime} 30^{\prime \prime N}-83^{\circ} 57^{\prime} \mathrm{W}, \mathrm{m} 2350$, 8.VI.1997, leg. Anderson R., oak forest litter 97-008D, 1 ex. (paratype, CNCo); S. José/Cart., km 68, Int. Amer. Hwy., nr. Tres de Junio, 09³9’46"N-8350'59"W, m 2560, 18.VI.1998, leg. Anderson R., wet cloud for. litter 98-101C, 1 ex. (paratype, cNCo); S. José/ Cart., km 72, Int. Amer. Hwy., Ojo de Agua 3 km W., 09³7’30"N-8350’30"W, m 2950, 7.VI.1997, leg. Anderson R., oak for. litter 97-005A, 1 ex. (paratype, CNCO).

\section{CEPHALOCYCLUS DURANGOENSIS (BATES, 1887)}

(Figs. 10-12)

Aphodius hogei var. durangoensis Bates, 1887: 91; Blackwelder, 1944: 212.

Aphodius (Platyderus) durangoensis; Schmidt A., 1913: 123 (as a synonym of hogei).

Aphodius (Platyderides) durangoensis; Schmidt A., 1922: 49 (as a synonym of hogei); Dellacasa M., 1988: 123 .

Aphodius pugil Balthasar, 1946: 55 (new synonymy).

Cephalocyclus durangoensis; Dellacasa M. \& alii, 1998: 142 (doubtful new status and new combination); Dellacasa M. \& alii, 2002: 215 (lectotype designation, new status and new combination).

Type locality: Refugio in Durango [Estado de Durango, Mexico].

Type repository: Natural History Museum. London.

Redescription: length 6.0-7.0 mm; oval-elongate, moderately convex, shiny; nearly glabrous. Brownish; epistoma, disc of pronotum and elytral suture darker. Head transverse with epistoma feebly gibbous at centre, finely sparsely and subregularly punctured, punctation somewhat irregular and coarser distally; clypeus anteriorly subtruncate, widely rounded at sides; distinctly bordered, edge elongately and sparsely ciliate laterally; genae acutely angulate, posteriorly truncate, elongately and sparsely ciliate, protruding much more than eyes; frontal suture extremely fine and faintly trigibbous; front sparsely and subregularly punctured. Pronotum widely transverse, moderately convex, distinctly flattened on sides, finely and sparsely punctured, punctation sparser on disc, denser on sides; latter rounded and with cerciniform edge sparsely and elongately ciliate; hind angles obliquely truncate; base bisinuate, not bordered. Scutellum superficially microreticulate, sparsely but rather coarsely punctured. Elytra subparallel-sided, moderately convex, on preapical declivity with short and sparse yellowish hairs; epipleura wide, sparsely and elongately ciliate; striae fine, subcrenulate; interstices nearly flat, finely microreticulate, superficially and sparsely punctured. Hind tibiae superior apical spur as long as first tarsomere; latter nearly as long as the following three combined. Male: head larger, relatively less convex; genae strongly protruding outward; pronotum more transverse and less convex; fore tibiae apical spur stout, strong, apically acuminate and abruptly bent downward; aedeagus fig. 12. Female: head smaller, relatively more convex; genae less protruding outward; pronotum subquadrate, relatively more convex; fore tibiae apical spur slender, straightly frontward directed.

Collecting notes: found in cattle and horse dung.

Distribution: Mexico (Coahuila, Durango, Michoacán, Nuevo León, Oaxaca). 
Remarks: on the base of the male holotype specimen of Aphodius (Platyderides) pugil Balthasar, 1946 preserved in the National Museum of Prague, we establish the name as a junior synonym of Cephalocyclus durangoensis (Bates, 1887).

Material examined: MexICo - Coahuila: 33 mi. SE Saltillo, nr. Jame, 7500 ft., 10.VII.1963, leg. Howden H. \& A., 3 exx. (CNCO); Durango: Ciudad Juarez, El Salto, 18.IX.1961, leg. Reyes P., 5 exx. (CNCO); carr. Mex 40-El Salto, Coscomate, m 2100, 2342’21.5"N-105³4’31.1"W, 20.VII.2004, leg. Dellacasa M. \& Martínez I., 5 exx. (DCG); km 125 carr. El Salto-Mazatlán, El Salto dint. W, m 2400, $23^{\circ} 45^{\prime} 20.6^{\prime \prime N}-105^{\circ} 31^{\prime} 17.0^{\prime \prime} \mathrm{W}, 20 . \mathrm{VII} .2004$, leg. Dellacasa M. \& Martínez I. (horse dung), 373 exx. (DCG); Durango, 5 mi. W, 6500 ft., 12.VII.1964, leg. Martin J. E. H., 2 exx. (CNCO); Durango, 7 mi. W, 7000 ft., 26.VII.1964, leg. McGuffin W. C., 1 ex. (CNCO); El Salto, 24 mi. E, 7500 ft., 15.VII.1964, leg. Martin J. E. H., 3 exx. (CNCO); El Salto, 25 mi. E, 17.VII.1964, leg. Howden H. F., 2 exx. (CNCO); El Salto, 28 mi. E, 22.VII.1964, leg. Howden H. F., 2 exx. (CNCO); El Salto, 11 mi. SW, 22.VII.1964, leg. Howden H. F., 1 ex. (CNCO); El Salto, 3 mi. W, 19.VI.1964, leg. Howden H. F., 2 exx. (CNCO); idem, 11.VII.1964, leg. Howden H. F., 13 exx. (CNCO); El Salto, 7 mi. W, 29.VI.1964, leg. Howden H. F., 1 ex. (CNCO); El Salto, 10 mi. W, 14.VI.1964, leg. Howden H. F., 1 ex. (CNCO); idem, 19.VI.1964, leg. Howden H. F., 11 exx. (CNCO); idem, 26.VI.1964, leg. Howden H. F., 2 exx. (CNCO); idem, 27.VI.1964, leg. Howden H. F., 1 ex. (CNCO); idem, 29.VI.1964, leg. Howden H. F., 1 ex. (CNCO); idem, 30.VI.1964, leg. Howden H. F., 1 ex. (CNCO); idem, 1.VII.1964, leg. Howden H. F., 3 exx. (CNCO); idem, 2.VII.1964, leg. Howden H. F., 38 exx. (CNCO); idem, 3.VII.1964, leg. Howden H. F., 1 ex. (cNCO); idem, 4.VII.1964, leg. Howden H. F., 2 exx. (CNCO); idem, 5.VII.1964, leg. Howden H. F., 20 exx. (CNCO); idem, 10.VII.1964, leg. Howden H. F., 9 exx. (CNCO); idem, 11.VII.1964, leg. Howden H. F., 9 exx. (CNCO); idem, 12.VII.1964, leg. Howden H. F., 1 ex. (CNCO); idem, 15.VII.1964, leg. Howden H. F., 1 ex. (CNCO); idem, 17.VII.1964, leg. Howden H. F., 3 exx. (CNCO); idem, 18.VII.1964, leg. Howden H. F., 3 exx. (CNCO); idem, 30.VII.1964, leg. Howden H. F., 1 ex. (CNCO); idem, 9000 ft., 17.VI.1964, leg. Martin J. E. H., 6 exx. (CNCO); idem, 19.VI.1964, leg. Howden H. F., 1 ex. (CNCO); idem, 25.VI.1964, leg. Martin J. E. H., 2 exx. (CNCO); idem, 29.VI.1964, leg. Martin J. E. H., 7 exx. (CNCO); idem, 20.VI-6.VII.1964, leg. Martin J. E. H., 395 exx. (CNCO); idem, 1.VII.1964, leg. Martin J. E. H., 22 exx. (cNCO); idem, 5.VII.1964, leg. McGuffin W. C., 17 exx. (CNCO); idem, 7.VII.1964, leg. McGuffin W. C., 49 exx. (CNCO); idem, 8.VII.1964, leg. McGuffin W. C., 72 exx. (CNCO); idem, 8.VII.1964, leg. Martin J. E. H., 134 exx. (CNCO); idem, 9.VII.1964, leg. Martin J. E. H., 218 exx. (CNCO); idem, 10.VII.1964, leg. McGuffin W. C., 33 exx. (CNCO); idem, 11.VII.1964, leg. McGuffin W. C., 49 exx. (CNCO); idem, 11.VII.1964, leg. Martin J. E. H., 64 exx. (CNCO); idem, 12.VII.1964, leg. Martin J. E. H., 110 exx. (CNCO); idem, 12.VII.1964, leg. McGuffin W. C., 77 exx. (CNCO); idem, 15.VII.1964, leg. McGuffin W. C., 7 exx. (CNCO); idem, 15.VII.1964, leg. Martin J. E. H., 59 exx. (CNCO); idem, 16.VII.1964, leg. McGuffin W. C., 83 exx. (cNCo); idem, 17.VII.1964, leg. Martin J. E. H., 214 exx. (CNCO); idem, 18.VII.1964, leg. McGuffin W. C., 60 exx. (CNCO); idem, 19.VII.1964, leg. McGuffin W. C., 12 exx. (CNCO); idem, 19.VII.1964, leg. Martin J. E. H., 94 exx. (CNCO); idem, 20.VII.1964, leg. McGuffin W.C., 5 exx. (CNCO); idem, 21.VII.1964, leg. McGuffin W. C., 17 exx. (CNCO); idem, 22.VII.1964, leg. McGuffin W. C., 16 exx. (CNCO); idem, 23.VII.1964, leg. McGuffin W. C., 7 exx. (CNCO); idem, 24.VII.1964, leg. McGuffin W. C., 12 exx. (CNCO); idem, 25.VII.1964, leg. McGuffin W. C., 20 exx. (CNCO); idem, 1.VIII.1964, leg. Martin J. E. H., 192 exx. (CNCO); idem, 1.VIII.1964, leg. Martin J. E. H., 1 ex. (usNmw); idem, 3.VIII.1964, leg. McGuffin W. C., 3 exx. (cNCO); idem, 3.VIII.1964, leg. Martin J. E. H., 10 exx. (CNCO); idem, 4.VIII.1964, leg. McGuffin W. C., 6 exx. (CNCO); idem, 5.VIII.1964, leg. McGuffin W. C., 15 exx. (cNco); idem, 6.VIII.1964, leg. McGuffin W.C., 30 exx. (cNCo); idem, 7.VIII.1964, 
leg. McGuffin W. C., 3 exx. (cNCO); idem, 8.VIII.1964, leg. McGuffin W. C., 1 ex. (CNCO); idem, 9.VIII.1964, leg. McGuffin W. C., 24 exx. (cNco); idem, 10.VIII.1964, leg. McGuffin W. C., 2 exx. (CNCO); idem, 11.VIII.1964, leg. Martin J. E. H., 7 exx. (CNCO); idem, 11.VIII.1964, leg. McGuffin W. C., 6 exx. (CNCO); El Salto, 10 mi. W, 8800 ft., 18.VII.1964, leg. Powell J., 1 ex. (EMEB); El Salto, 20 Km W, 2.VIII.1998, leg. Caterino M. S., 7 exx. (EMEB); El Salto, 35 mi. W, 23.VII.1964, leg. Howden H. F., 1 ex. (CNCO); El Salto, 37 mi. W, P. Buenos Aires, 1.VII.1964, leg. Howden H. F., 2 exx. (cNCO); La Ciudad, 10 Km E, 2.VIII.1998, leg. Caterino M. S., 6 exx. (EMEB); La Ciudad, 9 mi. E, 9000 ft., 20.VII.1964, leg. Powell J., 1 ex. (Емев); La Michilía, 4/6.VII.1986, leg. Terron R., 3 exx. (DCG); Refugio, leg. Hoge, 1 ex.


104¹9'50.1"W, 2.VII.2004, leg. Huerta C. \& Anduaga S. (bosque pino encino), 3 exx. (DCG); Res. Biosf. La Michilía, R.cho de la Peña, 6.VII.1979, leg. Zunino M., 4 exx. (DCG); Michoacán: Cruz Gorda dint., m 2250, $19^{\circ} 21^{\prime} 58.5^{\prime \prime N}-101^{\circ} 29^{\prime} 33.1$ "W, 25.VII.2003, leg. Dellacasa M. \& Martínez I., 6 exx. (DCG, MSNTC); Nuevo León: Cerro Potosí, 10300 ft., 15-16.VII.1963, leg. Howden H. \& A., 23 exx. (CNCO); Oaxaca: Llano de las Flores, Aserradero Atepec, $\mathrm{m}$ 1600, 17²26’37.1"N-96³0’12.2"W, 30.VI.2003, leg. Martínez I., Suarez T. \& Zunino M., 331 exx. (DCG, MSNTC); Praderero «Atepec», 2500 m, $17^{\circ} 26^{\prime} 36.5 \mathrm{~N}-96^{\circ} 30^{\prime} 11.2 \mathrm{~W}, 10 . \mathrm{VII} .2002$, leg. Dellacasa M. \& Martínez I., 6 exx. (MSNTC).

\section{CEPHALOCYCLUS FULIGINOSUS (HAROLD, 1863)}

(Figs. 13-15, 64)

Aphodius fuliginosus Harold, 1863: 330; Bates, 1887: 90; Blackwelder, 1944: 212.

Aphodius (Platyderus) fuliginosus; Schmidt A., 1913: 123.

Aphodius (Platyderides) fuliginosus; Schmidt A., 1922: 47; Dellacasa M., 1988: 132.

Cephalocyclus fuliginosus; Dellacasa M. \& alii, 1998: 142 (lectotype designation and new combination); Deloya \& Ibáñez-Bernal, 2000: 322; Dellacasa G. \& alii, 2001: 114; Dellacasa M. \& alii, 2002: 212 .

Type locality: Oaxaca, Mexico.

Type repository: Muséum national d'Histoire naturelle. Paris.

Redescription: length 5.0-7.0 mm; feebly convex, subshiny, elytra dull. Dark piceous, sometimes elytra paler. Head with epistoma feebly convex, distinctly microreticulate, finely sparsely rather irregularly punctured; clypeus subsinuate at middle, widely rounded at sides, thinly bordered, edge feebly upturned and elongately bristled; genae distinct, ciliate, more protruding than eyes; frontal suture distinct, faintly gibbous at middle and at sides. Pronotum widely transverse, with trace of longitudinal impression on disc, feebly convex, faintly flattened on sides, distinctly microreticulate, finely and very sparsely punctured; sides nearly faintly pubescent, bordered, edge sparsely bristled; hind angles obtusely rounded; base distinctly bisinuate, not bordered. Scutellum elongate, flat, distinctly microreticulate, with few superficial punctures on base only. Elytra oval-elongate, feebly widened toward apex, finely striate; striae superficially punctured, subcrenulate; interstices flat, strongly microreticulate, sericeous; sparsely, irregularly and finely punctured, sparsely shortly pubescent toward apex; epipleura distinct, elongately bristled on basal three quarters; shoulder not denticulate. Hind tibiae superior apical spur as long as tarsomere; latter as long as the following three combined. Male: head widely transverse, genae sharply angulated, frontal suture relatively more distinct and raised; fore tibiae relatively more slender and elongate, their apical spur relatively stout 
and abruptly bent downward; mesotarsomeres distinctly plumped (fig. 61), the first as long as the following three combined, latters of equal length and apically subdentiform, the fifth plumped; claws stout, superior one flattened and subdenticulate at base; aedeagus fig. 15. Female: head relatively less transverse, genae obtusely rounded, frontal suture nearly faint; epistoma much feebly gibbous; pronotum relatively narrowed frontward; fore tibiae relatively shorter and stouter; their apical spur slender and elongate, regularly straight; mesotarsomeres and claws normally shaped.

Collecting notes: found in dung of cattle, horse, and sheep dung, also taken at white and black lights.

Distribution: Guatemala, Mexico (Chihuahua, Distrito Federal, Durango, Guanajuato, Hidalgo, Jalisco, Edo. de México, Michoacán, Morelos, Nayarit, Oaxaca, Puebla, Sinaloa, Tlaxcala, Zacatecas).

Material examined: MExICo - Distrito Federal: San Angel, 18.VI.1961, leg. Janzen D. H., 1 exx. (EMEB); Chihuahua: Arroyo Mesteno, Sierra del Nido, 7600 ft., 11.VII.1959, leg. Russell W. C., 5 exx. (ЕМЕB); idem, 15.VII.1959, leg. Russell W. C., 1 ex. (ЕMEB); idem, 21.VII.1959, leg. Russell W. C., 3 exx. (EMEB); Basaseachic, 28¹3'00.4"N-108¹3'27.5"W, m 2037, 26.VI.2005, leg. Dellacasa M. \& Martínez I., 104 exx. (DCG, MSNTC); Cañon Prieto nr. Primavera, 6500-6800 ft., 2.VII.1947, leg. Cazier, D. Rockefeller Exp., 5 exx. (AMNHNY); idem, 2.VII.1947, leg. Gertsch, D. Rockefeller Exp., 1 ex. (AMNHNY); Catarinas, 5800 ft., 25.VII.1947, leg. Gertsch, D. Rockefeller Exp., 1 ex. (AMNHNY); idem, 25.VII.1947, leg. Michener, D. Rockefeller Exp., 4 exx. (AMNHNY); idem, 25.VII.1947, leg. Spieth, D. Rockefeller Exp., 2 exx. (AMNHNY); 5 mi. N Cerro Campana, Sierra del Nido, 5600 ft., 28.VI-1.VII.1959, leg. Russell W. C., 1 ex. (EMEB); idem, 6-8.VII.1959, leg. Russell W. C., 2 exx. (EMEB); Cuauhtemoc 15 mi. E, 6600 ft., 11.VII.1964, leg. Chemsak J. A. \& Powell J. (black \& white lights), 48 exx. (EMEB); Hidalgo del Parral 25 mi. W, 6800 ft., 14.VII.1964, leg. Chemsak J.A. \& Powell J. (black \& white lights), 21 exx. (EMEB); idem, 15.VII.1964, leg. Chemsak J.A. \& Powell J. (black \& white lights), 1 ex. (EMEB); Huejotitán, 5700 ft., 21.VII.1947, leg. Michener, D. Rockefeller Exp., 1 ex. (AMNHNY); Huerachic dint., 2806’25.9"N-10800’29.4"W, m 2258, 26.VI.2005, leg. Dellacasa M. \& Martínez I., 7 exx. (MSNTC); Llana de Río Santa Clara, 27 mi. W. Parrita, 12.VIII.1950, leg. R. F. Smith, 1 ex. (AMNHNY); Madera, 7200 ft., 6.VII.1947, leg. Michener, D. Rockefeller Exp., 2 exx. (AMNHNY); Matachic, 7.VII.1947, leg. Cazier, D. Rockefeller Exp., 3 exx. (AMNHNY); Matachic, 2 mi. W, 6400 ft., 7.VII.1947, leg. Cazier, D. Rockefeller Exp., 8 exx. (AMNHNY); idem, 7.VII.1947, leg. Michener, D. Rockefeller Exp., 2 exx. (AMNHNY); Matachic, 8 mi. W, 7200 ft., 8.VII.1947, leg. Cazier, D. Rockefeller Exp., 5 exx. (AMNHNY); idem, 8.VII.1947, leg. Gertsch, D. Rockefeller Exp., 3 exx. (AMNHNY); idem, 8.VII.1947, leg. Schramel, D. Rockefeller Exp., 3 ex. (AMNHNY); idem, 8.VII.1947, leg. Spieth, D. Rockefeller Exp., 2 exx. (AMnhNy); P.N. Barranca del Cobre, San Rafael dint., 27³0’34.2"N-10752’08.5"W, m 2150, 25.VI.2005, leg. Dellacasa M. \& Martínez I., 3 exx. (MSNTC); San José Babicora, 5.VII.1947, leg. Cazier, D. Rockefeller Exp., 6 exx. (AMNHNY); idem, 5.VII.1947, leg. Gertsch, D. Rockefeller Exp., 17 exx. (AMNHNY); idem, 5.VII.1947, leg. Michener, D. Rockefeller Exp., 33 exx. (AMNHNY); Santa Barbara, 63 mi. W, 5500 ft., 20.VII.1947, leg. Gertsch, D. Rockefeller Exp., 1 ex. (AMNHNY); idem, 20.VII.1947, leg. Schramel, D. Rockefeller Exp., 1 ex. (AMNHNY); Santa Barbara Dist., Santa Barbara, 6300 ft., 17.VII.1947, leg. Cazier, D. Rockefeller Exp., 1 ex. (AMNHNY); idem, 18.VII.1947, leg. Cazier, D. Rockefeller Exp., 1 ex. (AMNHNY); idem, 18.VII.1947, leg. Spieth, D. Rockefeller Exp., 2 exx. (AMNHNY); Santa Barbara Dist., Santa Barbara, 7500 ft., 18.VII.1947, leg. Gertsch, D. RockefellerExp., 4 exx. (AMNHNY); idem, 18.VII.1947, leg. Spieth, D. Rockefeller Exp., 1 ex. (AMNHNY); Durango: 18 de Marzo, m 1650, 2349’24.4"N-104²6’25.6"W, 18.VII.2004, leg. Dellacasa M. \& Martínez I., 1 ex. (DCG); Durango, 26.5 mi. SW, 21.VII.1982, leg. F. G. 
Dellacasa et al.: Revision of genus Cephalocyclus and ten new species
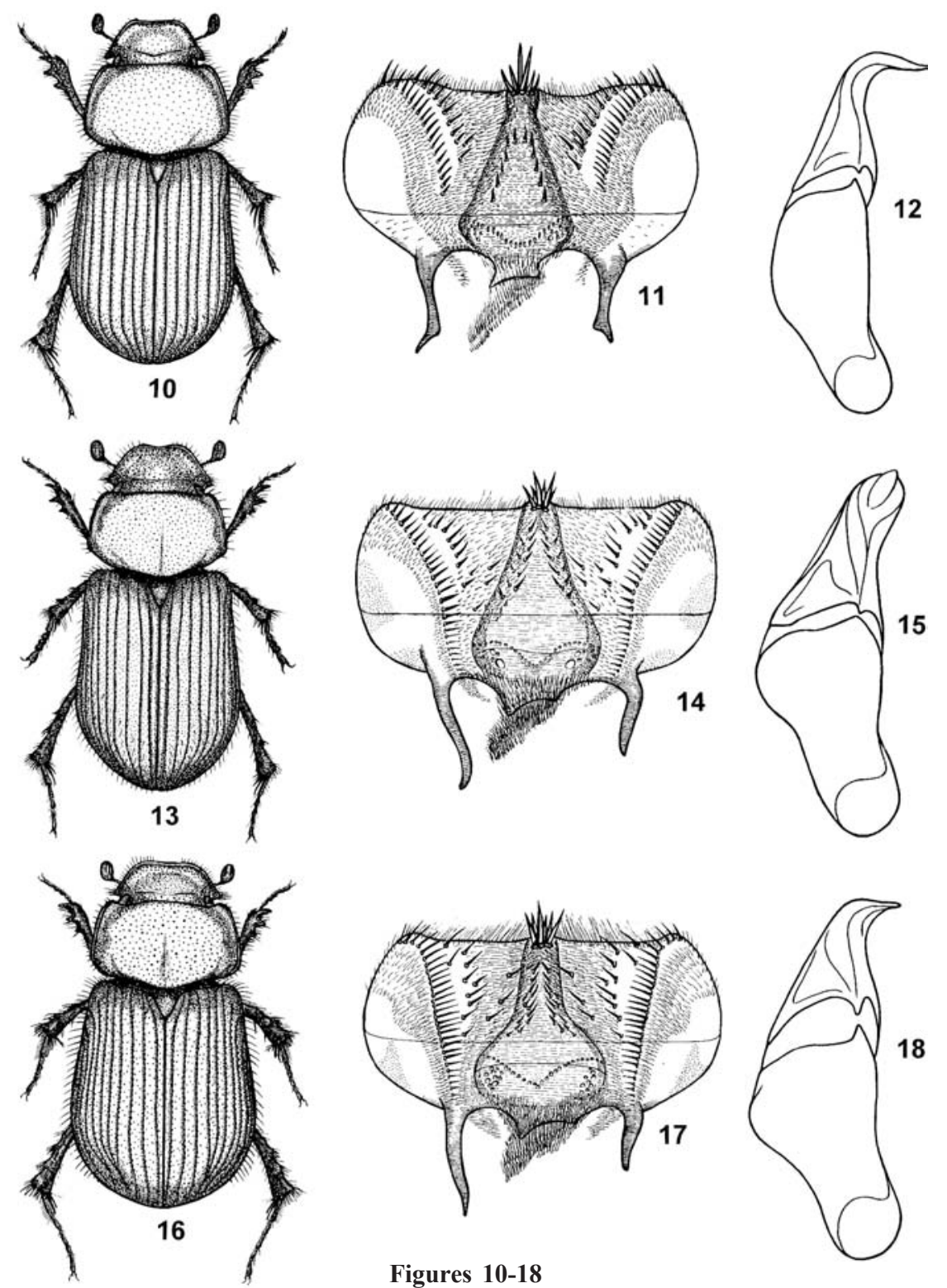

10-12.- Cephalocyclus durangoensis: 10.- habitus of male (length $6.0 \mathrm{~mm}$; Refugio in Durango, Edo. Durango, Mexico); 11.- epipharynx; 12.- aedeagus (lateral view); 13-15.- Cephalocyclus fuliginosus: 13.habitus of male (length $6.0 \mathrm{~mm}$; Rancho el Alemán, Reserva de la Biosfera «La Michilía», Edo. Durango, Mexico); 14.- epipharynx; 15.- aedeagus (lateral view); 16-18.- Cephalocyclus gravidus: 16.- habitus of male (length $7.0 \mathrm{~mm}$; carr. Valle de Bravo, Temescaltepec, Edo. México, Mexico); 17.- epipharynx; 18.aedeagus (lateral view). 
Andrews (blacklight), 86 exx. (CSCAG); Durango, 30 mi. W, Tepalcates, 17.VI.1964, leg. Howden H. F., 144 exx. (CNCO); Coyotes, 8300 ft., 8.VIII.1947, leg. Cazier, D. Rockefeller Exp., 1 ex. (AMNHNY); El Salto, 6 mi. NE, 8500 ft., 10.VIII.1947, leg. Michener, D. Rockefeller Exp., 1 ex. (AMNHNY); El Salto, 21 mi. E, 18.VII.1964, leg. Chemsak J. A., 2 exx. (EMEB); Encino, 6200 ft., 27.VII.1947, leg. Gertsch, D. Rockefeller Exp., 1 ex. (AMNHNY); idem, 27.VII.1947, leg. Schramel, D. Rockefeller Exp., 8 exx. (amnhny); idem, 27.VII.1947, leg. Spieth, D. Rockefeller Exp., 3 exx. (AMnhNy); La Michilía, leg. Anduaga, 3 exx. (DCG); La Michilía, Rancho Alemán, 3.VII.1979, leg. Zunino M., 63 exx. (DCG); idem, 6.VII.1979, leg. Zunino M., 4 exx. (DCG); Minillas, Res. Biosf. La Michilía, 8.VIII.1994, leg.

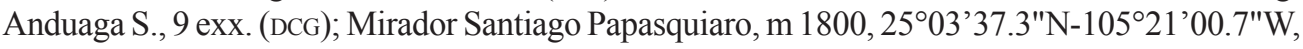
17.VII.2004, leg. Dellacasa M. \& Martínez I. (cow dung), 57 exx. (DCG); Nueva Patria [Santo

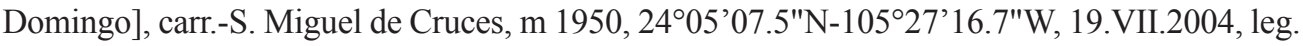
Dellacasa M. \& Martínez I. (bosque pino-encino, horse dung), 6 exx. (DCG); Otinapa dint., $\mathrm{m} 1200$, $24^{\circ} 03^{\prime} 26.5^{\prime \prime N}-105^{\circ} 00^{\prime} 29.6^{\prime \prime W}, 19 . V I I .2004$, leg. Dellacasa M. \& Martínez I., 4 exx. (DCG); Otinapa, 8200 ft., 11.VIII.1947, leg. Cazier, D. Rockefeller Exp., 2 exx. (AMnHNY); Palos Colorados, 8000 ft., 5.VIII.1947, leg. Cazier, D. Rockefeller Exp., 8 exx. (AMNHNY); idem, 5.VIII.1947, leg. Michener, D. Rockefeller Exp., 1 ex. (Amnhny); Res. Biosf. La Michilía, El Alemán, m 2250, 2320¹5"N104¹0'13"W, 30.VI.2004, leg. Huerta C. \& Anduaga S., 2 exx. (DCG); Tepalcates, 30 mi. W Durango, 17.VI.1964, leg. Howden H. F., 1 ex. (USNMw); Hidalgo: Ajacuba, 21.VI.1937, leg. Cartwright O. L., 1 ex. (UsNMw); Las Sabanillas, Llano Grande, 20¹0’44.8"N-9842’44.6"W, m 2935, 4.VII.2005, leg. Dellacasa M. \& Martínez I., 15 exx. (MSNTC); Mineral del Chico, 20¹3’00.3"N-9843'51.1"W, m 2358, 4.VII.2005, leg. Dellacasa M. \& Martínez I., 19 exx. (MSNTC); Mineral del Chico, El Oyamel, $20^{\circ} 10^{\prime} 31.3^{\prime \prime N}-98^{\circ} 42^{\prime} 92.5^{\prime \prime W}, \mathrm{~m} 2887,29$.VI.2006, leg. Dellacasa M., Fresi C. \& Martínez I., horse dung, 2 exx. (DCG); Jalisco: Chapala 6 mi. W, 30.VI.1963, leg. Doyen J., 1 ex. (ЕMEB); Edo. de México: Aculco env., 2002’08.6"N-9951'05.3"W, m 2615, 7.VII.2006, leg. Dellacasa M., Fresi C. \& Martínez I., 106 exx. (DCG, MSNTC); Amecameca, 8.VI.1897, leg. Koebele, 1 ex. (uSNMw); Carr. Valle de Bravo, Temascaltepec, 4.VI.1979, leg. Zunino M., 1 ex. (DCG); Centro Cerimonial Otomí dint., m 2650, 19³1’24.8"N-99³2'14.1"W, 23.VII.2004, leg. Dellacasa M. \& Martínez I. (sterco ovino), 9 exx. (DCG); Pyramids, 9.VI.1935, leg. Embury M. A., 4 exx. (AMNHNY); Michoacán: Los Azufres, 1949`54.8"N-100³9’40.7"W, m 2916, 2.VII.2005, leg. Dellacasa M. \& Martínez I., 12 exx. (MSNTC); Macho de Agua, Zitácuaro, 23.VI.1979, leg. Zunino M., 1 ex. (DCG); Maravatío, 20.VI.1979, leg. Zunino M., 7 exx. (DCG); Morelia 34 mi. E, 2.VII.1963, leg. Foster A., 1 ex. (EMEB); Panindicuaro, 1958'14.1"N-101²4’09.1"W, m 1860, 29.VI.2005, leg. Dellacasa M. \& Martínez I., 3 exx. (MSNTC); Quiroga dint., $19^{\circ} 40^{\prime} 05.2^{\prime \prime} \mathrm{N}-101^{\circ} 32^{\prime} 08.0^{\prime \prime} \mathrm{W}, \mathrm{m} 2105$, 1.VII.2005, leg. Dellacasa M. \& Martínez I., 3 exx. (MSNTC); San Andrés Tzirondaro, 19³9’44.8"N-101³8'13.2"W, m 2090, 1.VII.2005, leg. Dellacasa M. \& Martínez I., 3 exx. (MSNTC); Tuxpan, 11.VII.1951, leg. Hurd P. D., 3 exx. (EMEB); Nayarit: Santa María del Oro, m 1000, 1.VI.1902, leg. Hoffmann C., 1 ex. (IEx); Puebla: 17 mi. S Puebla, 27.VI.1957, leg. Chemsak J. A. \& Rannells B. J., 1 ex. (ЕмEB); Sinaloa: Mazatlán 5 mi. N, 24.VII.1964, leg. Chemsak J. A. \& Powell J. (black \& white lights), 1 ex. (ЕMEB); Tlaxcala: Tlaxco, 2.VI.1979, leg. Zunino M., 3 exx. (DCG); Zacatecas: 3 km desp. Jerez de García Salinas, Sustihuacán dint., m 1750, 22³6’49.8"N-10308'42.6"W, 15.VII.2004, leg. Dellacasa M. \& Martínez I. (horse dung), 1 ex. (DCG); El Refugio dint., m 1950, 22 ${ }^{\circ} 55^{\prime} 29.9^{\prime \prime} \mathrm{N}-103^{\circ} 07^{\prime} 13.8^{\prime \prime} \mathrm{W}, 15 . V I I .2004$, leg. Dellacasa M. \& Martínez I. (horse dung), 2 exx. (DCG) 
Dellacasa et al.: Revision of genus Cephalocyclus and ten new species

\section{CEPHALOCYCLUS GRAVIDUS (HAROLD, 1863)}

(Figs. 16-18)

Aphodius gravidus Harold, 1863: 330; Bates, 1887: 91: Blackwelder, 1944: 212.

Aphodius pinguescens Harold, 1871: 261 (nomen nudum); Dellacasa M. \& alii; 2002: 218 (lectotype designation and as a synonym of gravidus).

Aphodius (Platyderus) gravidus; Schmidt A., 1913: 123.

Aphodius (Platyderides) gravidus; Schmidt A., 1922: 50; Dellacasa M., 1988: 136.

Cephalocyclus gravidus; Dellacasa M. \& alii, 1998: 144 (lectotype designation and new combination); Deloya \& Ibáñez-Bernal, 2000: 323; Dellacasa M. \& alii, 2002: 218.

Type locality: Mexico.

Type repository: Muséum national d'Histoire naturelle. Paris.

Redescription: length 6.0-8.0 mm; convex, moderately shiny, nearly glabrous, piceous, sides of pronotum, elytra and legs paler, antennal club yellowish. Head transverse, with epistoma feebly gibbous at middle, superficially microreticulate, sparsely regularly distinctly punctured; clypeus subtruncate, sparsely and elongately bristled at sides, bordered, edge cerciniform; frontal suture faint, feebly trigibbous; genae acutely angulated, posteriorly truncate, elongately and sparsely bristled, protruding much more than eyes. Pronotum transverse, moderately convex, distinctly flattened at sides, superficially microreticulated, with trace of longitudinal median impression, sparsely regularly very finely punctured; sides weakly rounded, strongly bordered; rather shortly and sparsely bristled; hind angles broadly rounded; base bisinuate, not bordered. Scutellum finely microreticulate, irregularly punctured at base. Elytra broadly oval, convex; striae fine, distinctly impressed, superficially punctured, subcrenulate; interstices feebly convex, distinctly microreticulate, very sparsely and finely punctured; laterally and on preapical declivity with short and sparse yellowish hairs; shoulder not denticulate; epipleurae wide, elongately and sparsely bristled on basal three quarters. Hind tibiae superior apical spur as long as first tarsomere; latter nearly equal to the following three combined. Male: head large, relatively less convex; genae strongly protruding outward; pronotum strongly transverse, relatively less convex; fore tibiae with apical spur stout, strong, apically acuminate, outwardly directed and abruptly bent downward; aedeagus fig. 18. Female: head relatively more narrow, more convex; genae less protruding outward; pronotum subquadrate, relatively more strongly convex; fore tibiae with apical spur apically regularly acuminate, anteriorly directed.

Collecting notes: found in cattle and horse dung.

Distribution: Mexico (Chihuahua, Distrito Federal, Durango, Hidalgo, Jalisco, Edo. de México, Michoacán).

Material examined: Mexico - Chihuahua: Basaseachic, 28 ${ }^{\circ} 13^{\prime} 00.4^{\prime \prime} \mathrm{N}-108^{\circ} 13^{\prime} 27.5^{\prime \prime} \mathrm{W}$, m 2037, 26.VI.2005, leg. Dellacasa M. \& Martínez I., 4 exx. (DCG); Distrito Federal: Contreras, 30.V.1946, leg. Pallister J. \& D., 12 exx. (AmnhnY); Distrito Federal, Mexico City, 1946, leg. Pallister J. \& D., 1 ex. (Amnhny); Durango: Mesa Larga, Res. Biosf. La Michilía, Cuiyachapa, 22.VII.1988, leg. Anduaga S., 3 exx. (DCG); Piedra Herrada, Res. Biosf. La Michilía, 23.VII.1989, leg. Anduaga S., 3 exx. (DCG); idem, 9.VIII.1989, leg. Anduaga S., 3 exx. (DCG); idem, 14.VIII.1989, leg. Anduaga S., 2 exx. (DCG); Santa Martha, 3 km ante, m 2700, 1904'58.1"N- 
9943’48.2"W, 25.VII.2004, leg. Dellacasa M. \& Martínez I. (horse dung), 4 exx. (DCG); Hidalgo: Zacualtipán, leg. Hoge, 1 ex. (DCG); Jalisco: Sierra Manantlán, 4/5.VIII.1990, 9 exx. (DCG); Edo. de México: Aculco env., 2002'08.6"N-99 51'05.3"W, m 2615, 7.VII.2006, leg. Dellacasa M., Fresi C. \& Martínez I., 1 ex. (DCG); Chapultepec, 2450 m, 20.V.1994, leg. Scheer, 4 exx. (ВАСв); Coyotepec, $19^{\circ} 04^{\prime} 35.4^{\prime \prime} \mathrm{N}-99^{\circ} 23^{\prime} 48.3^{\prime \prime} \mathrm{W}, \mathrm{m} 2831$, 4.VII.2005, leg. Cabrero F. \& Trotta N., 13 exx. (MSNTC, DCG); Laguna de Zempoala, $1^{\circ} 05^{\prime} 08^{\prime \prime N}-99^{\circ} 18^{\prime} 58^{\prime \prime} \mathrm{W}, \mathrm{m} 2800$, 4.VII.2005, leg. Cabrero F. \& Trotta N., 9 exx. (MSNTC); carr. Santa Martha-Zempoala, 1904’34.4"N99²1’41.1"W, m 3048, 8.VII.2006, leg. Dellacasa M., Fresi C. \& Martínez I., 12 exx. (DCG); Rancheria San Antonio, Carr. Valle de Bravo, 27.VII.2001, leg. Dellacasa M. \& Martínez I., 2 exx. (DCG); idem, 29.VII.2001, leg. Dellacasa M. \& Martínez I., 2 exx. (DCG); rd. Mex-15 Zitácuaro-Toluca, El Jacal, m 2300, $19^{\circ} 24^{\prime} 13.1 \mathrm{~N}-100^{\circ} 05^{\prime} 53.9 \mathrm{~W}, 27$.VII.2001, leg. Dellacasa M., 2 exx. (MSNTC); Real de Arriba, Temescaltepec, VII.1933, 2 exx. (USNmw); Santa Martha, 24.V.1964, leg. Halffter G., 1 ex. (usNmw); Michoacán: Cruz Gorda dint., 19²2’44.6"N$101^{\circ} 29^{\prime} 47.3^{\prime \prime W}, \mathrm{~m} 2706,30 . V I .2005$, leg. Dellacasa M. \& Martínez I., 21 exx. (MSNTC, DCG); Diablo de Zirahuén, $19^{\circ} 22^{\prime} 26^{\prime \prime N}-101^{\circ} 48^{\prime} 28^{\prime \prime} \mathrm{W}, \mathrm{m}$ 2384, 30.VI.2005, leg. Dellacasa M. \& Martínez I., 1 ex. (MSNTC); Los Azufres, 1949’54.8"N-100³9’40.7"W, m 2916, 2.VII.2005, leg. Dellacasa M. \& Martínez I., 4 exx. (mSNTC); Macho de Agua, Zitácuaro, 23.VI.1979, leg. Zunino M., 2 exx. (USNmw); Michoacán, 1.VI.1946, leg. Foshag W. F., 2 exx. (USNMw); PátzcuaroTacámbaro, Santa Clara del Cobre, 25.VII.2001, leg. Dellacasa M. \& Martínez I., 2 exx. (DCG); Zitácuaro, leg. Anduaga S., 5 exx. (DCG)

\section{CEPHALOCYCLUS HALFFTERI SP. NOV. (Figs. 19-21)}

Type locality: carretera Tlatlauquitepec-Zapotitlán Tablas, Crucero el 12, m 2200, 17²2 '56.2 N, 9850’16.7" W, Guerrero, Mexico.

Type repository: Dellacasa Collection, Genoa (Italy).

Description: length 6.0-7.0 mm; short oval, convex; head, pronotum and scutellum moderately shiny in males, dull in females; elytra strongly microreticulate thus dull, very shortly and sparsely pubescent on preapical declivity. Piceous; clypeal margin, pronotum sides and elytra somewhat paler; legs piceous; antennal club blackish. Head with epistoma faintly convex on disc, superficially microreticulate, finely and somewhat irregularly punctured, punctation coarser and denser distally; clypeus truncate anteriorly, widely rounded laterally, thickly bordered, the edge feebly upturned and sparsely ciliate laterally; genae acutely angulate, elongately ciliate, strongly protruding more than eyes; frontal suture obsolete; front very finely and regularly punctured. Pronotum transverse, moderately convex, feebly flattened laterally toward anterior and posterior angles only, very finely, almost sparsely and regularly punctured; sides feebly curved, thickly bordered, edge glabrous; hind angles obtusely rounded; base distinctly bisinuate, not bordered. Scutellum somewhat depressed laterally, faintly carinate at middle, sparsely punctured. Elytra oval, widened posteriorly, rather convex; finely striate; striae superficially punctured, not crenulate; interstices flat, strongly microreticulate, without any trace of punctation; epipleural margin sparsely elongately ciliate till more than the half of length. Hind tibiae superior apical spur somewhat shorter than first tarsomere; latter as long as the following three combined. 
Male: head and pronotum shiny, relatively larger and less convex; fore tibiae apical spur stout and abruptly downward bent; aedeagus fig. 21. Female: head and pronotum dull, relatively narrower and more convex; fore tibiae apical spur slender and straight.

Collecting notes: collected in horse dung.

Distribution: Mexico (Guerrero, Edo. de México).

Derivatio nominis: named in honour of professor Gonzalo Halffter, eminent entomologist in Jalapa (Veracruz, Mexico).

Type material: MEXICO - Guerrero: carretera Tlatlauquitepec-Zapotitlán Tablas, Crucero el 12, m 2200, 17²8'56.2 N, 98 50’16.7" W, 23.VII.2003, leg. Dellacasa M. \& Martínez I., 11 exx. (holotype male, allotype female, paratypes; DCG); Edo. de México: Entrada Parque Nacl. Popocatepetl, m 2600, 24.VII.2004, leg. Dellacasa M. \& Martínez I., horse dung, 1 ex. (paratype, DCG); Río Frío dint., 19²1’00"N-9840’10"W, 3.VI.2005, leg. Cabrero F. \& Trotta N., 4 exx. (paratypes, DCG).

\section{CEPHALOCYCLUS HOGEI (BATES, 1887)}

(Figs. 22-24)

Aphodius hogei Bates, 1887: 90; Blackwelder, 1944: 212.

Aphodius (Platyderus) hogei; Schmidt A., 1913: 123.

Aphodius (Platyderides) hogei; Schmidt A., 1922: 49; Dellacasa M., 1988: 140.

Aphodius transversus Robinson, 1940: 145 (new synonymy).

Cephalocyclus hogei; Dellacasa M. \& alii, 1988: 142 (doubtful new combination); Dellacasa M. \& alii, 2002: 213 (lectotype designation and new combination).

Type locality: Las Vigas, Mexico.

Type repository: Natural History Museum. London.

Redescription: length 4.0-6.0 mm; oblong, moderately convex, nearly glabrous; head and pronotum rather shiny, elytra almost dull. Piceous, elytra brownish. Head with epistoma faintly convex, microreticulate, sparsely, finely and somewhat irregularly punctured; clypeus subtruncate anteriorly, widely rounded at sides, quite thickly bordered, edge rather elongately bristled laterally; genae acute, posteriorly truncate, elongately ciliate, strongly protruding more than eyes; frontal suture finely impressed; front sparsely and superficially punctured. Pronotum widely transverse, feebly convex, distinctly flattened on sides, minutely reticulate, finely, superficially and sparsely punctured; sides thickly bordered, edge elongately ciliate; hind angles obtusely truncate; base bisinute, not bordered. Scutellum strongly microreticulate, sparsely punctured. Elytra elongate, feebly widened toward apex and with short sparse hairs on preapical declivity, deeply striate; striae rather superficially punctured, subcrenulate; interstices moderately convex, strongly microreticulate, sparsely punctured; epipleura distinct and elongately ciliate. Hind tibiae superior apical spur as long as first tarsomere; latter as long as the following three combined. Male: head relatively more large and transverse; genae sharply angulate; pronotum relatively more transverse; fore tibiae more slender and elongate with the apical spur stout and abruptly bent downward; aedeagus fig. 24. Female: head relatively smaller and less transverse; genae less angulate and 
relatively less protruding; pronotum narrower frontward; fore tibiae shorter and stouter with apical spur slender, elongate and straight.

Collecting notes: taken from dung of cattle, horse, and sheep.

Distribution: Guatemala, Mexico (Coahuila, Distrito Federal, Durango, Hidalgo, Edo. de México, Michoacán, Morelos, Oaxaca, Puebla, Querétaro, Veracruz), U.S.A. (Arizona)?

Remarks: examination of the male holotype specimen of Aphodius transversus Robinson (1940) preserved in the Smithsonian Institution, Washington, DC, revealed that is was a synonym of Cephalocyclus hogei (Bates, 1887). The male holotype is the only specimen of Aphodius transversus known, and it is remarkable that no other specimen has been taken from such a relatively well collected area as Southern Arizona. Several Mexican species belong in this genus, and the holotype of Aphodius transversus may be an incorrectly labeled specimen actually collected in Mexico.

Material examined: Mexico - Coahuila: Rancho San Francisco, 25²3’28.5"N-100³7'16.7"W, m 2220, 3.VII.2006, leg. Dellacasa M., Fresi C. \& Martínez I., horse dung, 1 ex. (DCG); Durango: La Michilía, Rancho La Peña, 6.VII.1979, leg. Zunino M., 34 exx. (DCG); Refugio, leg. Hoge, 1 ex. (DCG);

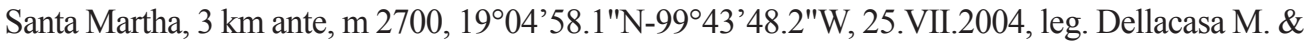
Martínez I. (horse dung), 22 exx. (DCG); Hidalgo: Las Sabanillas, Llano Grande, 20¹0'44.8"N9842’44.6"W, m 2935, 4.VII.2005, leg. Dellacasa M. \& Martínez I., 2 exx. (MSNTC); Mineral del Chico, El Oyamel, $20^{\circ} 10^{\prime} 31.3^{\prime \prime N}-98^{\circ} 42^{\prime} 92.5^{\prime \prime W}, \mathrm{~m} 2887,29 . V I .2006$, leg. Dellacasa M., Fresi C. \& Martínez I., horse dung, 58 exx. (DCG, MSNTC); Edo. de México: carr. Lagunas de Zempoala-Santa Martha, 1904'19.4"N-99²0'29.4"W, m 3127, leg. Cabrero F. \& Trotta N., 4 exx. (MSNTC); carr. Santa MarthaZempoala, 1904'34.4"N-99²1’41.1"W, m 3048, 8.VII.2006, leg. Dellacasa M., Fresi C. \& Martínez I., 138 exx. (DCG, MSNTC); carr. Temoaya-Jiquipilco, m 3000, 19³2’09.9"N-99²9’47.3"W, 23.VII.2004, leg. Dellacasa M. \& Martínez I., 13 exx. (DCG); carr--Valle de Bravo, Rancheria San Antonio, m 2550, $19^{\circ} 19^{\prime} \mathrm{N}-100^{\circ} 05^{\prime} \mathrm{W}, 27 . \mathrm{VII} .2001$, leg. Dellacasa M. \& Martínez I., 22 exx. (MSNTC, DCG); Centro Cerimonial Otomí dint., m 2650, 19³1’24.8"N-99³2'14.1"W, 23.VII.2004, leg. Dellacasa M. \& Martínez I. (sheep dung), 74 exx. (DCG); El Guarda, m 2800, 27.VII.1969, 1 ex. (DCG); La Marquesa, 20.VI.1979, leg. Zunino M., 14 exx. (DCG); Paso de Cortes, m 3300, 1905’11.4"N-98³8’47.1"W, 24.VII.2004, leg. Dellacasa M. \& Martínez I. (cow dung), 71 exx. (DCG); Raices env., 1909`44.9"N-9948’17.9"W, m 3499, 8.VII.2006, leg. Dellacasa M., Fresi C. \& Martínez I., horse dung, 6 exx. (DCG); Real de Arriba, Temescaltepec, VIII.1933, leg. Hinton H. E. \& Usinger R. L., 1 ex. (USNMw); Río Frío env., 19²0’37.7"N9840’28.8"W, m 2991, 8.VII.2005, leg. Dellacasa M., Martínez I., Cabrero F. \& Trotta N., 18 exx. (DCG); Salazar, 28.VI.1954, leg. Halffter G., 2 exx. (USNMw); Michoacán: Cruz Gorda dint., 19²2’44.6"N101²9’47.3"W, m 2706, 30.VI.2005, leg. Dellacasa M. \& Martínez I., 271 exx. (DCG, MSNTC); Los Azufres, 1949'54.8"N-100³9'40.7"W, m 2916, 2.VII.2005, leg. Dellacasa M. \& Martínez I., 224 exx. (MSNTC); idem, m 2550, 1949'57.2"N-100³9'42.2"W, 22.VII.2004, leg. Dellacasa M. \& Martínez I., 98 exx. (DCG); rd Mex-41 Pátzcuaro-Tacámbaro, La Estacada, m 2450, 19²4'N-101³2W, 25.VII.2001, leg. Dellacasa M. \& Martínez I., 15 exx. (MSNTC); Oaxaca: km 133 carr. Tuxtepec-Oaxaca, Atepec, Llano de las Flores, $\mathrm{m} 2600,1^{\circ} 26^{\prime} 37.1^{\prime \prime N}-96^{\circ} 30^{\prime} 13.2^{\prime \prime} \mathrm{W}, 3 . V I I .2004$, leg. Martínez I. \& Zunino M. (horse dung), 1 ex. (DCG); idem, 11.VIII.2003, leg. Martínez I., Reyes P. \& Suarez T., 1 ex. (DCG); Puebla: San Diego Texmelucan env., Mpio. Chalchicomula, 1901'07"N-97²7’21"W, m 2572, 27.VI.2006, leg. Dellacasa M. \& Fresi C., 1 ex. (DCG); carr. San Nicolas de los Ranchos-Paso de Cortes, $19^{\circ} 05^{\prime} 50.5^{\prime \prime} \mathrm{N}-98^{\circ} 36^{\prime} 51.0^{\prime \prime} \mathrm{W}, \mathrm{m} 3350,10$. VII.2006, leg. Dellacasa M., Fresi C. \& Martínez I., cattle dung, 6 exx. (DCG); Santa Cruz Cuoutomatitlán, 4.VII.2002, leg. Aragon A., 2 exx. (MSNTC); 
Dellacasa et al.: Revision of genus Cephalocyclus and ten new species
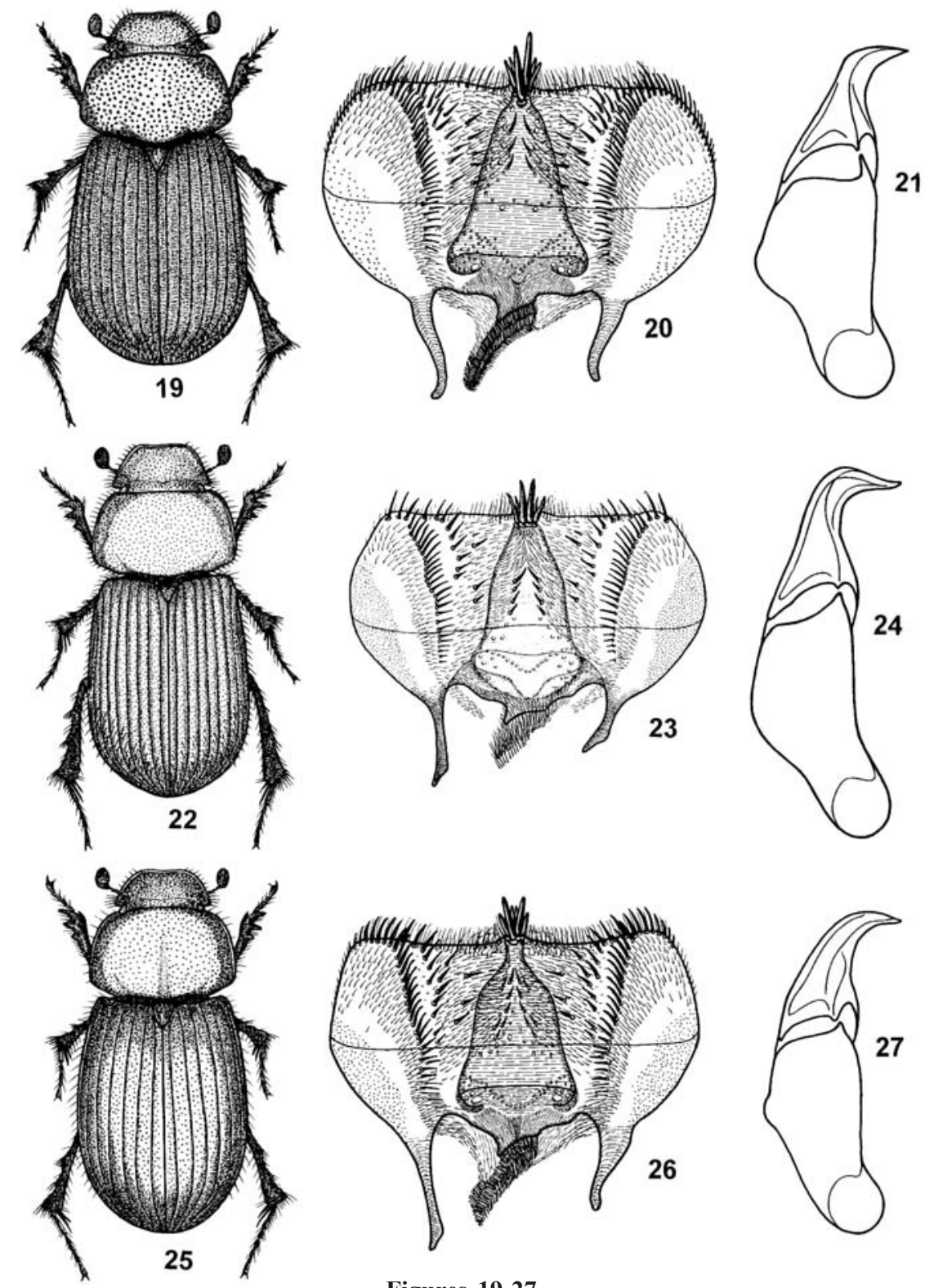

Figures 19-27

19-21.- Cephalocyclus halffteri: 19.- habitus of male (length $7.0 \mathrm{~mm}$; carr. Tlatlauquitepec-Zapotitlán Tablas, Crucero el 12, Edo. Guerrero, Mexico); 20.- epipharynx; 21.- aedeagus (lateral view); 22-24.Cephalocyclus hogei: 22.- habitus of male (length $5.0 \mathrm{~mm}$; Real de Arriba, Edo. México, Mexico); 23.epipharynx; 24.- aedeagus (lateral view); 25-27.- Cephalocyclus howdenorum: 25.- habitus of male (length 8.0 mm; Cerro Potosí, Edo. Nuevo León, Mexico); 26.- epipharynx; 27.- aedeagus (lateral view). 
Querétaro: La Vuelta dint., Parador Santa Martha, m 1550, $21^{\circ} 16^{\prime} 09.2 \mathrm{~N}-99^{\circ} 09^{\prime} 50.4 \mathrm{~W}, 19 . \mathrm{VII} .2001$, leg. Dellacasa M. \& Martínez I., 3 exx. (MSNTC); rd. Pinal de Amoles- Peña Blanca, La Quebrada, m 2300, $21^{\circ} 07^{\prime}$ N-99 38 W, 20.VII.2001, leg. Dellacasa M. \& Martínez I., 4 exx. (MSNTC); Veracruz: carr. Ayahualulco-Ixhuacán de los Reyes, 17.VI.2002, leg. Martínez I., \& Suarez T., 2 exx. (MSNTC); Coscomatepec, Cuiyachapa, 12.V.1998, leg. Cruz M., 15 exx. (DCG); idem, 14.VI.1999, leg. Alvarado M\&Alii, 188 exx. (DCG); idem, 13.VII.1998, leg. Alvarado M. \& Cruz M., 60 exx. (MSNTC); idem, m 2700, 13.VII.1998, leg. Cruz M \& Alii, 160 exx. (DCG); Estación las Lajas, camino, 13.VI.1994, leg. Arellano \& Sanchez, 6 exx. (DCG); Las Vigas, 5500 ft., 30.VI.1941, leg. Dybas H. S., 4 exx. (USNMw); Las Vigas, leg. Hoege 1 ex. (paratype) (USNMw); Perote dint., San José Aguazuelas, 18.VII.2000, leg. Pensado T., Santiago Q \& Suarez T., 27 exx. (MSNTC); idem, en la «Y», 2.VII.2001, leg. Suarez T., 33 exx. (MSNTC); idem, m 2800, 17.VI.2002, leg. Dellacasa M., Martínez I. \& Suarez T., 21 exx. (MSNTC); idem, $19^{\circ} 25^{\prime} 06.9$ N-97 $12^{\prime} 40.6 \mathrm{~W}, 2$.VII.2001, leg. Dellacasa M., 369 exx. (DCG, MSNTC).

\section{CEPHALOCYCLUS HOWDENORUM SP. NOV.}

(Figs. 25-27)

Type locality: Cerro Potosí, 10300', Nuevo León, Mexico.

Type repository: Canadian National Collection. Ottawa.

Description: length 7.0-8.0 mm, broadly oval-elongate, strongly convex, shiny, glabrous. Dark piceous; clypeal margin and pronotal sides somewhat paler, elytra dark brownish-yellow; legs and antennal club brownish. Head with epistoma gibbous, very finely and superficially punctured at centre, punctation coarser and sparser toward clypeal margin, distally somewhat confuse; clypeus faintly sinuate at middle, rounded at sides, distinctly bordered, the edge not upturned and laterally elongately ciliate; genae obtusely rounded, elongately ciliate, protruding more than eyes; front evenly distinctly punctured. Pronotum feebly transverse, strongly convex, slightly flattened laterally, evenly and rather sparsely punctured, with a median longitudinal impunctate line; sides feebly arcuate, rather thickly bordered, edge sparsely ciliate; hind angles obtusely rounded, base feebly bisinuate, not bordered. Scutellum somewhat depressed at each side, punctured on basal half. Elytra strongly convex, broadly oval-elongate, not microreticulate so shiny, rather deeply striate; striae superficially punctured, not crenulate; interstices nearly flat, finely sparsely punctured; epipleural carina not denticulate at shoulder; epipleural margin elongately ciliate. Hind tibiae superior apical spur as long as first tarsomere; latter as long as the following three combined. Male: fore tibiae apical spur relatively stouter and more abruptly bent downward; head and pronotum more transverse, less convex, more sparsely and superficially punctured; aedeagus fig. 27 . Female: fore tibiae apical spur relatively slender, less abruptly downward bent; head and pronotum less narrowed frontwardly, more convex and more densely punctured.

Collecting notes: found in cattle dung.

Distribution: Mexico (Nuevo León).

Derivatio nominis: named in honour of Henry and Anne Howden (Ottawa, Canada), who first collected the taxon.

Type material: Mexıco - Nuevo León: Cerro Potosí, 10.300', 15-16.VII.1963, leg. Howden H. \& A., 51 exx. (holotype male, allotype female, paratypes; CNCO, HAHCO); idem, 12.100', 1.VI.1987, leg. Turnbow 
R., 32 exx. (paratypes; RTC, DCG); idem, m 3000, 18.VI.1972, leg. Halffter, 1 ex. (paratype; HAHCO); idem, Galeana, 10-12.000', 27.VI.1969, leg. Peck S. \& J., 23 exx. (paratypes; HAHCO); idem, m 3750, 4.VI.1983, leg. Kaulbars, 9 exx. (paratypes; HAHCO); idem, 4.VI.1983, leg. Peck S. \& J. (summit litter Ber), 1 ex. (paratype; нАнСо); Cerro El Potosí, 2452’28.1"N-100¹3’14.9"W, m 3274, 4.VII.2006, leg. Dellacasa M., Fresi C. \& Martínez I., cattle dung, 248 exx. (paratypes, DCG, MSNTC); Montemorelos, 13 mi. W, Chorros de Agua, 19.VI.1969, leg. Peck S., 5 exx. (paratypes; нAнCO); Monterreal env., 25¹3'35.8"N$100^{\circ} 24^{\prime} 40.2^{\prime \prime} \mathrm{W}, \mathrm{m} 2736$, 3.VII.2006, leg. Dellacasa M., Fresi C. \& Martínez I., horse dung, 8 exx. (paratypes, DCG); cerca de Peña Nevada, 16.VII.1964, leg. Rotger B., 3 exx. (paratypes; USNMw).

\section{CEPHALOCYCLUS LAGOI SP. NOV.}

(Figs. 28-30)

Type locality: Chapinque [ $=$ Chipinque] Mesa, m 2100, 20 km SE Saltillo. Coahuila, Mexico.

Type repository: University of Mississippi, Department of Biology, University (MS, USA).

Description: length 5.0-5.5 mm; elongate, convex, shiny, glabrous. Brownish; clypeal margin, pronotum sides and elytra reddish-brown; legs reddish, antennal club brownish. Head with epistoma faintly convex on disc, finely irregularly punctured, punctation somewhat coarser and denser distally; clypeus subtruncate anteriorly, widely rounded at sides, thinly bordered, the edge laterally elongately ciliate; genae rather acutely angulate, elongately ciliate, distinctly protruding more than eyes; frontal suture distinct, somewhat raised; front very sparsely, finely and regularly punctured. Pronotum moderately transverse, convex, finely and regularly almost irregularly punctured; puntation finer and sparser on disc, relatively coarser and denser on sides and toward the basal margin; sides near subparallel, distinctly bordered, the edge sparsely ciliate; hind angles obtuse; base bisinuate, not bordered. Scutellum faintly convex, densely punctured laterally and basally. Elytra oval elongate, slightly widened posteriorly; striae superficial, wide, subcrenulate; interstices feebly convex, superficially microreticulate, very finely and sparsely punctured. Hind tibiae superior apical spur as long as first tarsomere; latter nearly as long as the following three combined. Male: head relatively larger, less convex and more sparsely punctured with genae strongly protruding more than eyes; pronotum somewhat more transverse and less convex; fore tibiae apical spur abruptly downward bent and outward curved; aedeagus fig. 30 . Female: head relatively smaller, more convex, and more densely punctured with genae less strongly protruding more than eyes; pronotum, narrower frontward and more convex; fore tibiae apical spur straight.

Collecting notes: found in cattle and horse dung.

Distribution: Mexico (Coahuila, Nuevo León, Querétaro, San Luis Potosí).

Derivation nominis: named in honour of Paul K. Lago, curator in Department of Biology, University of Mississippi (USA).

Type material: Mexico - Coahuila: Chapinque [=Chipinque] Mesa, $20 \mathrm{~km}$ SE Saltillo, $\mathrm{m} 2100$, 7.VII.1992, leg. Lago P. K., 1 ex. (holotype male; DBumu); idem, m 2100, 7.VII.1992, leg. Lago P. K., 3 exx. (allotype female, paratypes; DBUMU, DCG); 20 mi. S.E. Saltillo, Rt 015, 6000', 20-21.VI.1971,

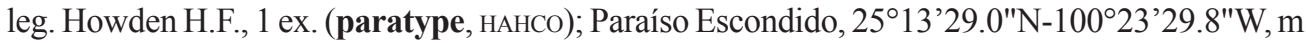
2476, 5.VII.2006, leg. Dellacasa M., Fresi C. \& Martínez I., 2 exx. (paratypes, DCG); Puerto MexicoSaltillo, carr. libre-Saltillo, $25^{\circ} 14^{\prime} 52.6^{\prime \prime} \mathrm{N}-100^{\circ} 56^{\prime} 57.1^{\prime \prime} \mathrm{W}, \mathrm{m} 2256,1$.VII.2006, leg. Dellacasa M., 
Fresi C. \& Martínez I., 409 exx. (paratypes, DCG, MSNTC); Nuevo León: hacia Cerro El Potosí, $24^{\circ} 53^{\prime} 15.8^{\prime \prime N}-100^{\circ} 11^{\prime} 52.2^{\prime \prime W}, \mathrm{~m} 2313$, 4.VII.2006, leg. Dellacasa M., Fresi C. \& Martínez I., cow dung, 50 exx. (paratypes, DCG); Cerro El Potosí, 24² 52’28.1"N-100¹3'14.9"W, m 3274, 4.VII.2006, leg. Dellacasa M., Fresi C. \& Martínez I., cattle dung, 21 exx. (paratypes, DCG); Monterreal env., $25^{\circ} 13^{\prime} 35.8^{\prime \prime N}-100^{\circ} 24^{\prime} 40.2^{\prime \prime} \mathrm{W}, \mathrm{m} 2736,3 . V I I .2006$, leg. Dellacasa M., Fresi C. \& Martínez I., horse dung, 6 exx. (paratypes, DCG); Querétaro: Xilitla, 40 Km W, $m$ 1700, 12.VI-6.VIII.1983, leg. Peck S. \& J., 1 ex. (paratype, нансо); San Luis Potosí: Real de Catorce, El Potrero, 234'30.2"N$100^{\circ} 50^{\prime} 58.3^{\prime \prime W}, \mathrm{~m} 2456,6$.VII.2006, leg. Dellacasa M., Fresi C. \& Martínez I., horse dung, 121 exx. (paratypes, DCG, MSNTC).

\section{CEPHALOCYCLUS LURIDIVENTRIS (HAROLD, 1862)}

(Figs. 31-33, 67)

Aphodius luridiventris Harold, 1862: 385; Bates, 1887: 92; Blackwelder, 1944: 213.

Aphodius (Platyderus) luridiventris; Schmidt A., 1913: 123.

Aphodius (Platyderides) luridiventris; Schmidt A., 1922: 50; Dellacasa M., 1988: 156.

Cephalocyclus luridiventris; Dellacasa M. \& alii, 1998: 147 (lectotype designation and new combination); Deloya \& Ibáñez-Bernal, 2000: 323; Dellacasa M. \& alii, 2002: 221.

Type locality: Parada [Edo. Oaxaca], Mexico.

Type repository: Muséum national d'Histoire naturelle. Paris.

Redescription: length 7.0-8.0 mm; oblong, feebly convex, moderately shiny. Piceous, antennal club dark testaceous, legs testaceous-reddish. Head with epistoma faintly gibbous at middle, distinctly coarsely punctured toward apical margin, nearly smooth at center, posteriorly and laterally coarsely irregularly punctured; clypeus anteriorly nearly truncate, widely rounded and elongately bristled at sides, quite strongly bordered, edge upturned; genae acutely angulate, elongately bristled, protruding much more than eyes; frontal suture nearly faint. Pronotum transverse, moderately convex, slightly flattened at sides, sparsely superficially irregularly punctured, glabrous; lateral margins feebly rounded, distinctly bordered, edge elongately bristled; hind angles obtusely rounded; base bisinuate, not bordered. Scutellum indistinctly microreticulate, superficially sparsely punctured. Elytra feebly widened posteriorly, distinctly striate; striae very superficially punctured, not crenulate; interstices almost flat, indistinctly microreticulate, very superficially sparsely punctured; shoulder not denticulate. Hind tibiae superior apical spur slightly longer than first tarsomere; latter shorter than following three combined. Claws stout, angulately broadened at base. Male: head and pronotum relatively less convex, less densely and less coarsely punctured; fore tibiae relatively more slender and elongate, apical spur abruptly bent downward; middle tibiae with inferior apical spur spatulate, outward curved (fig. 67); aedeagus fig. 33. Female: head and pronotum relatively more convex, much densely and much coarsely punctured; fore tibiae relatively shorter and more robust, apical spur straight; middle tibiae with inferior apical spur shortened, robust, outwardly curved and apically rounded.

Collecting notes: none.

Distribution: Mexico (Distrito Federal, Oaxaca, Puebla).

Material examined: Mexico - Puebla: Cinco Señores, Sallé coll., 1 ex. (Usnmw). 
Dellacasa et al.: Revision of genus Cephalocyclus and ten new species
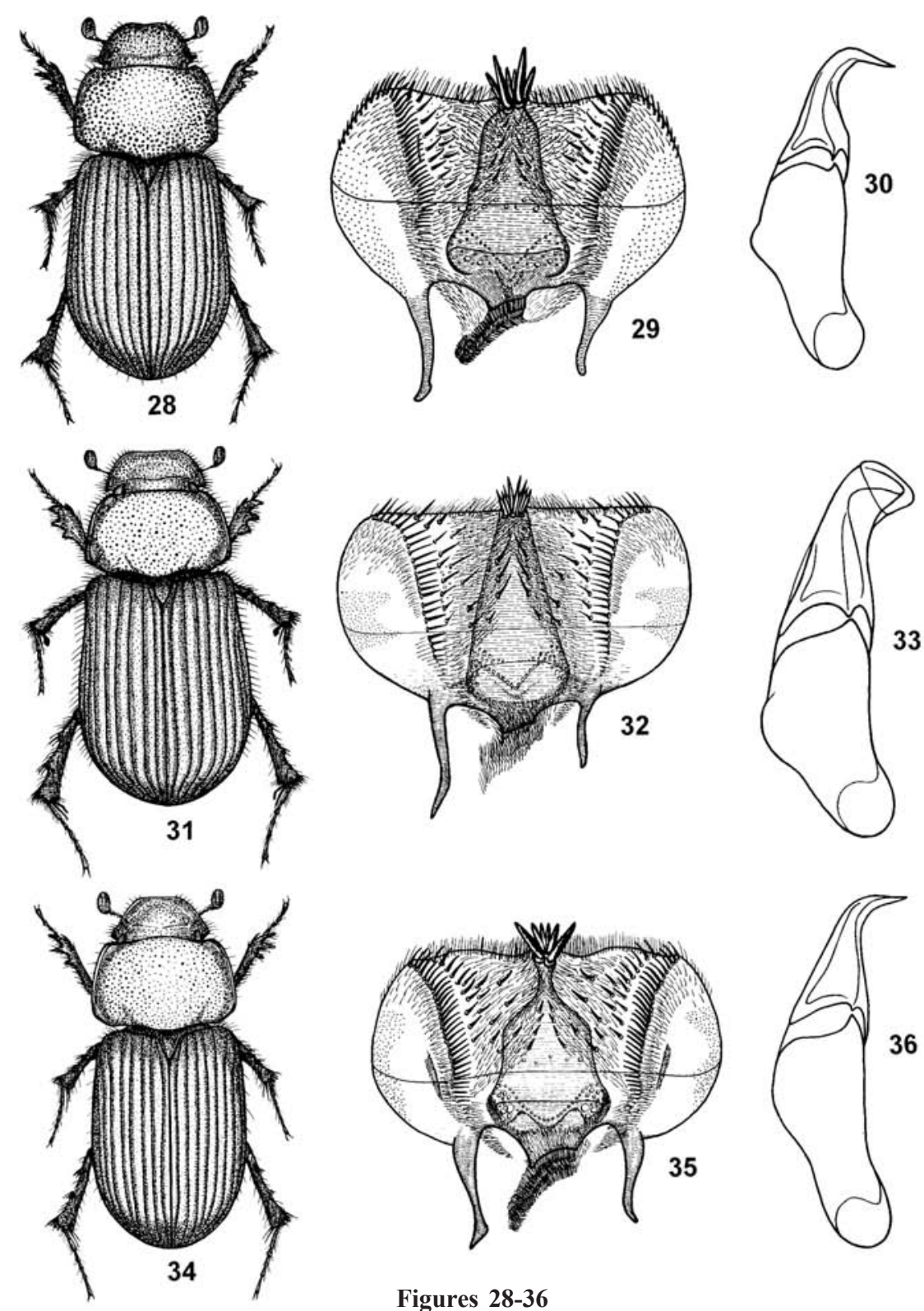

Figures 28-36

28-30.- Cephalocyclus lagoi: 28.- habitus of male (length $5.0 \mathrm{~mm}$; Chapinque [=Chipinque] Mesa, Edo. Coahuila, Mexico); 29.- epipharynx; 30.- aedeagus (lateral view); 31-33.- Cephalocyclus luridiventris: 31.habitus of male (length 8.0 mm; La Parada, Edo. Oaxaca, Mexico); 32.- epipharynx; 33.- aedeagus (lateral view); 34-36.- Cephalocyclus luteolus: 34.- habitus of male (length $5.0 \mathrm{~mm}$; Silver City, Grant Co., New Mexico, U.S.A.); 35.- epipharynx; 36.- aedeagus (lateral view). 


\section{CEPHALOCYCLUS LUTEOLUS (HORN, 1887) COMB. NOV.}

(Figs. 34-36)

Aphodius luteolus Horn, 1887: 40; Leng, 1920: 250.

Aphodius (Platyderus) luteolus; Schmidt A., 1913: 123.

Aphodius (Platyderides) luteolus; Schmidt A., 1922: 48; Dellacasa M., 1988: 156.

Type locality: N.[ew] M.[exico].

Type repository: United States National Museum, Washington.

Redescription: length 5.0-6.0 mm; moderately elongate, subdepressed, shiny, glabrous. Yellowish-testaceous; head, pronotum and juxtasutural interstices darker; legs testaceous; antennal club yellowish. Head with epistoma flat, very sparsely and finely punctured; punctation denser and coarser distally; clypeus subtruncate, widely rounded at sides, quite thickly bordered, edge laterally elongately ciliate; genae angulate, elongately ciliate, strongly protruding more than eyes; frontal suture feebly raised, not tuberculate at all; front finely and subregularly punctured. Pronotum transverse, finely alutaceous, moderately convex, laterally feebly explanate, distinctly and irregularly punctured; punctation somewhat coarser and denser on sides; latter subparallel, distinctly bordered, edge almost thin, rather elongately and sparsely ciliate; hind angles obliquely truncate; base bisinuate, not bordered. Scutellum microreticulate, distinctly and sparsely punctured on basal half. Elytra moderately elongate, subparallel-sided, not denticulate at shoulder, finely but rather deeply striate; striae crenulate; interstices moderately convex, sparsely punctulate; epipleural margin thick, elongately and sparsely ciliate on basal two thirds. Superior apical spur of hind tibiae somewhat longer then first tarsomere; latter as long as following three combined. Male: head relatively more transverse and less convex; genae acutely angulate and strongly protruding more than eyes; pronotum more transverse and more sparsely punctured; fore tibiae apical spur short, thick and abruptly downward curved; aedeagus fig. 36. Female: head relatively less transverse and more convex; genae obtusely angulate and less strongly protruding more than eyes; pronotum narrowed frontward, more convex and more densely punctured; fore tibiae apical spur slender, elongate and straight.

Collecting notes: taken at white and black lights, food preferences unknown.

Distribution: U. S. A. (Arizona, New Mexico, Texas, Utah).

Material examined: USA - New Mexico, Silver City, Grant Co., 15.VII.1973, leg. Lenczy C., 10 exx. (DCG).

\section{CEPHALOCYCLUS MEXICANUS (HAROLD, 1862)}

(Figs. 37-39, 65)

Aphodius mexicanus Harold, 1862: 380; Blackwelder, 1944: 213.

Aphodius omiltemius Bates, 1889: 392; Blackwelder, 1944: 213; Schmidt A., 1913: 172 (subgenere incerto); Dellacasa M., 1988: 172; Dellacasa M. \& alii, 2002: 211 (as synonym of mexicanus).

Aphodius (Platyderus) mexicanus; Schmidt A., 1913: 123.

Aphodius (Platyderides) mexicanus; Schmidt A., 1922: 49; Dellacasa M., 1988: 162.

Cephalocyclus mexicanus; Dellacasa M. \& alii, 1998: 148 (lectotype designation and new combination); Deloya, 1998: 101; Deloya \& Ibáñez-Bernal, 2000: 323; Dellacasa M. \& alii, 2002: 217. 
Type locality: Parada [Edo. Oaxaca], Mexico.

Type repository: Muséum national d'Histoire naturelle. Paris.

Redescription: length 5.0-6.0 mm; moderately convex, shiny, glabrous. Piceous; clypeal margin, pronotal sides and legs testaceous. Head with epistoma regularly convex, finely irregularly microreticulate, very finely and sparsely punctured; clypeus subtruncate, broadly rounded at sides and sparsely elongately bristled, bordered, edge anteriorly cerciniform; genae acutely angulate, posteriorly truncate, elongately sparsely bristled, distinctly protruding more than eyes; frontal suture not tuberculate. Pronotum transverse, indistinctly flattened at sides, moderately convex, finely sparsely punctured - punctation denser and stronger anteriorly and laterally -, very superficially microreticulate rather shiny, with trace of longitudinal median impression; sides strongly bordered, sparsely elongately bristled, feebly rounded, subsinuate before hind angles, latter obtusely rounded; base bisinuate, not bordered. Scutellum very superficially microreticulate, feebly convex at middle with some punctures irregularly scattered on sides. Elytra moderately convex, feebly widened at middle; striae fine, strongly impressed, indistinctly punctured; interstices moderately convex, near striae faintly microreticulate, almost smooth at middle so rather shiny, finely sparsely distinctly punctured; epipleurae strongly broadened, elongately sparsely bristled on basal two thirds; shoulder not denticulate. Hind tibiae superior apical spur slightly longer than first tarsomere; latter subequal to the following three combined. Male: head relatively more transverse, less convex, very superficially microreticulated and sparsely punctured; frontal suture nearly obsolete; genae relatively more angulate and more protruding; pronotum relatively more transverse and less convex, sides with double punctation, disc simply and superficially sparsely punctured; fore tibiae apical spur short, stout, apically acuminate, outward directed and abruptly bent downward; first mesotarsomere downwardly widened apically and therein with an exceptionally elongate apical seta, rather as long as the following three tarsomeres combined (fig. 62); aedeagus fig. 39. Female: head relatively narrower, more strongly convex, more distinctly microreticulate and punctured; frontal suture fine but distinct; genae less angulate and less protruding; pronotum narrowed frontward, more distinctly convex, with irregularly scattered double punctation; fore tibiae with apical spur slender, elongate, apically regularly acuminate, anteriorly directed.

Collecting notes: found in cattle and horse dung, and taken at white and black light.

Distribution: Guatemala, Mexico (Distrito Federal, Durango, Guerrero, Hidalgo, Jalisco, Edo. de México, Michoacán, Morelos, Nayarit, Oaxaca, Puebla, Querétaro, Sinaloa).

Material examined: Mexico - Durango: Buenos Aires, 7 mi. SW, 8800 ft., 20.VII.1982, leg. Andrews F. G. (cowdung), 3 exx. (CSCAG); Coyotes, 8300 ft., Durango Dist., 8.VIII.1947, leg. Cazier, D. Rockefeller Exp., 1 ex. (AMNHNY); Durango, 6200 ft., 14.VIII.1947, leg. Gertsch, D. Rockefeller Exp., 1 ex. (AMNhNY); Durango, 26.5 mi. SW, 21.VII.1982, leg. Andrews F. G. (blacklight), 3 exx. (CSCAG); El Salto, 12.VII.1990, leg. J. Stamatov, 2 exx. (AMNHNY); El Salto, 6 mi. N.E., 8500 ft., Durango Dist., 10.VIII.1947, leg. Cazier, D. Rockefeller Exp., 5 exx. (AMNHNY); idem, 10.VIII.1947, leg. Schramel, D. Rockefeller Exp., 1 ex. (AMNHNY); El Salto 28 mi. E, 8000 ft., 22.VII.1964, leg. Chemsak J. A. \& Powell J. (black \& white lights), 1 ex. (EMEB); km 125 carr. El Salto-Mazatlán, El

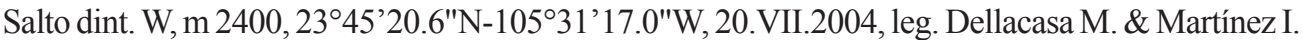
(horse dung), 18 exx. (DCG); Nueva Patria [Santo Domingo], carr.-S. Miguel de Cruces, $m$ 1950, $24^{\circ} 05^{\prime} 07.5^{\prime \prime} \mathrm{N}-105^{\circ} 27^{\prime} 16.7^{\prime \prime} \mathrm{W}, 19 . \mathrm{VII} .2004$, leg. Dellacasa M. \& Martínez I. (bosque pino-encino, 
horse dung), 159 exx. (DCG); Otinapa dint., $\mathrm{m} 1200,2^{\circ} 03^{\prime} 26.5^{\prime \prime} \mathrm{N}-105^{\circ} 00^{\prime} 29.6^{\prime \prime W}, 19 . V I I .2004$, leg. Dellacasa M. \& Martínez I., 61 exx. (DCG); Rancho la Peña, 20.VI.1979, leg. Zunino M., 3 exx. (DCG); Res. Biosf. La Michilía, El Alemán, m 2250, 23²0’15"N-104¹0’13"W, 30.VI.2004, leg. Huerta C. \& Anduaga S., 1 ex. (DCG); Guerrero: carr. Tlatlauquitepec-Zapotitlán Tablas, Crucero el 12, m 2200, 17²8’56.2"N-9850’16.7"W, 23.VII.2003, leg. Dellacasa M. \& Martínez I., 127 exx. (MSNTC, DCG); carr.-Olinalá, $1.5 \mathrm{~km}$ desp. Tres Caminos, $\mathrm{m} 1750,7^{\circ} 35^{\prime} 37^{\prime \prime} \mathrm{N}-98^{\circ} 44^{\prime} 19^{\prime \prime} \mathrm{W}, 22 . \mathrm{VII} .2003$, leg. Dellacasa M. \& Martínez I., 123 exx. (MSNTC, DCG); Hidalgo: Mineral del Chico, $20^{\circ} 13^{\prime} 00.3^{\prime \prime N}$ 98²3'51.1"W, m 2358, 4.VII.2005, leg. Dellacasa M. \& Martínez I., 4 exx. (MSNTC); Tejocotal dint., $20^{\circ} 08^{\prime} 07.5^{\prime \prime N}-98^{\circ} 09^{\prime} 08.6^{\prime \prime} \mathrm{W}, \mathrm{m} 2136,5 . \mathrm{VII} .2005$, leg. Dellacasa M. \& Martínez I., 228 exx. (MSNTC); Zacualtipán, leg. Hoge, 1 ex. (DCG); Jalisco: Concepción B. Aires, Paso Hondo, 12.VI.2003, leg.

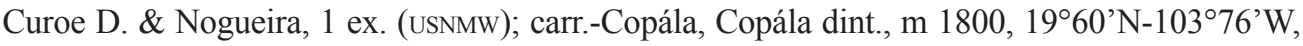
28.VII.2003, leg. Dellacasa M. \& Martínez I., 7 exx. (MSNTC, DCG); Puerto Los Mazos, 8 Km SE, Camino de Microondas, 19.VII.1994, leg. McCarty J. D., 1 ex. (EMEB); México: Aculco env., 2002’08.6"N-9951'05.3"W, m 2615, 7.VII.2006, leg. Dellacasa M., Fresi C. \& Martínez I., 68 exx. (DCG, MSNTC); carr.-Valle de Bravo, Rancheria San Antonio, $\mathrm{m} 2250,1^{\circ} 1^{\prime}{ }^{\prime} \mathrm{N}-100^{\circ} 05^{\prime} \mathrm{W}, 27 . \mathrm{VII} .2001$, leg. Dellacasa M. \& Martínez I., 66 exx. (MSNTC, DCG); Chalma, m 1550, 5.VII.1980, leg. Zunino M., 3 exx. (DCG); rd. Mex-15 Zitácuaro-Toluca, El Jacal, $\mathrm{m}$ 2300, 19²4’13.1N-10005'53.9W, 27.VII.2001, leg. Dellacasa M., 7 exx. (MSNTC); Real de Arriba, Temescaltepec, VII.1932, leg. Hinton H. E. \& Usinger R. L., 1 ex. (DCG); idem, VII.1933, leg. Hinton H. E. \& Usinger R. L., 1 ex. (USNmw); Tejupilco, Temescaltepec, VII.1932, 1 ex. (USNMw); Michoacán: Cruz Gorda dint., 19²2’44.6"N101²9'47.3"W, m 2706, 30.VI.2005, leg. Dellacasa M. \& Martínez I., 162 exx. (DCG, MSNTC); idem, m 2250, 19² $1^{\circ} 58.5^{\prime \prime N}-101^{\circ} 29^{\prime} 33.1^{\prime \prime W}, 25 . V I I .2003$, leg. Dellacasa M. \& Martínez I., 3 exx. (DCG); Los Azufres, 1949’54.8"N-100³9’40.7"W, m 2916, 2.VII.2005, leg. Dellacasa M. \& Martínez I., 40 exx. (MSNTC); idem, m 2550, 1949’57.2"N-100³9'42.2"W, 22.VII.2004, leg. Dellacasa M. \& Martínez I., 7 exx. (DCG); Patambán dint., m 1800, 19³8’19.8"N-102¹6’29.2"W, 26.VII.2003, leg. Dellacasa M. \& Martínez I., 2 exx. (MSNTC); rd. Mex-15 Morelia-Cd. Hidalgo, Mil Cumbres, m 2000, 19³9’N$100^{\circ} 59^{\prime}$ W, 26.VII.2001, leg. Dellacasa M. \& Martínez I., 1 ex. (MSNTC); rd. Mex-41 PátzcuaroTacámbaro, La Estacada, m 2450, 19²4'N-101²2'W, 25.VII.2001, leg. Dellacasa M. \& Martínez I., 7 exx. (DCG, MSNTC); rd. Mex-41 Pátzcuaro-Tacámbaro, Santa Clara del Cobre dint., m 2200, 19²4’N101³5’W, 25.VII.2001, leg. Dellacasa M. \& Martínez I., 1 ex. (MSNTC); Tacámbaro, 25.VII.2001, leg. Dellacasa M. \& Martínez I., 4 exx. (DCG); Uruapan, 28.11.1980, leg. Zunino M., 1 ex. (DCG); Nayarit: Ahuacatlán 6 mi. W, 19.VII.1954, leg. Schlinger E. I., 1 exx. (ЕмEB); Mirador Barranca del Oro, m 1650, 2059’35.0"N-104²7’56.9"W, 1.VIII.2003, leg. Dellacasa M. \& Martínez I., 8 exx. (MSNTC, DCG); Oaxaca: carr. Mex-135 ante Sola de Vega, $1900 \mathrm{~m}, 16^{\circ} 27^{\prime} 50.0^{\prime \prime} \mathrm{N}-96^{\circ} 59^{\prime} 27.9^{\prime \prime} \mathrm{W}$, 5.VII.2002, leg. Dellacasa M. \& Martínez I., 65 exx. (MSNTC); carr. Mex-135, Cieneguilla dint., 2000 m, 17²8'08.4"N-96 56'18.7"W, 9.VII.2002, leg. Dellacasa M. \& Martínez I., 2 exx. (MSNTC); El Cerezal, 36 Km NE Oaxaca, m 2300, 12.VI.1979, leg. Howden H. \& A., 1 ех. (нАнсо); Ixtlan, 14 mi. S, 7500', 27.VII.1971, leg. Newton A., 2 ex. (HAHCO); Mpio. San Mateo Río Hondo, El Manzanal, 2500 m, $16^{\circ} 07^{\prime} 57.4$ N-96 $29^{\prime} 48.5^{\prime \prime}$, 6.VII.2002, leg. Dellacasa M. \& Martínez I., 46 exx. (MSNTC); Oaxaca, 22 Km NE, m 2600, 12.VI.1979, leg. Howden H. \& A., 1 ex. (нАнсо); rd. Mex-135, San Gabriel Mixtepec dint., 2600 m, 16 $13^{\circ} 13.8^{\prime \prime N}-97^{\circ} 08^{\prime} 55.7^{\prime \prime W}, 9 . V I I .2002$, leg. Dellacasa M. \& Martínez I., 1 ex. (MSNTC); rd. Mex-175 Oaxaca-Tuxtepec, La Cumbre, 2500 m, 17¹0’31.2"N96³6’14.2"W, 10.VII.2002, leg. Dellacasa M. \& Martínez I., 4 exx. (MSNTC); rd. Mex-175-Miahuatlán, 
San José Pacifico, 2250 m, 1609’55.4N-96³0’07.1"W, 6.VII.2002, leg. Dellacasa M. \& Martínez I., 18 exx. (MSNTC); rd. Mitla-S. Pablo Ayutla, Laguna San Pablo, 1800 m, 1659’29.0"N-9607’41.0W, 7.VII.2002, leg. Dellacasa M. \& Martínez I., 10 exx. (mSNTC); San Pablo Ayutla dint., 1800 m, $17^{\circ} 01^{\prime} 06.1^{\prime \prime N}-96^{\circ} 05^{\prime} 54.8^{\prime \prime}$ W, 7.VII.2002, leg. Dellacasa M. \& Martínez I., 36 exx. (mSNTC); Sola de Vega, 5 Km S, m 1600, 4-9.VII.2005, leg. Curoe D., 1 ex. (USNMw); Suchixtepec, 8 Km S, Río Molino, m 2200, 16.VI.1979, leg. Howden H. \& A., 2 exx. (HAнсо); Puebla: Venta Grande, 2007’29.0"N$98^{\circ} 06^{\prime} 07.3 " \mathrm{~W}, \mathrm{~m} 2125,5 . \mathrm{VII} .2005$, leg. Dellacasa M. \& Martínez I., 39 exx. (MSNTC); Querétaro: La Vuelta dint., Parador Santa Martha, m 1550, $21^{\circ} 16^{\prime} 09.2$ N-99 $09^{\prime} 50.4$ W, 19.VII.2001, leg. Dellacasa M. \& Martínez I., 86 exx. (MSNTC, DCG); Landa de Matamoros, 17 mi. E, 5300', 28.VI.1971, leg. Newton A., 1 ex. (нансо); Pinal de Amoles, La Cañada, 2106' $57.3^{\prime \prime N}-99^{\circ} 39^{\prime} 47.9^{\prime \prime} \mathrm{W}, \mathrm{m}$ 2580, 3.VII.2005, leg. Dellacasa M. \& Martínez I., 22 exx. (DCG, MSNTC); rd. Cascada de Chuveje-San

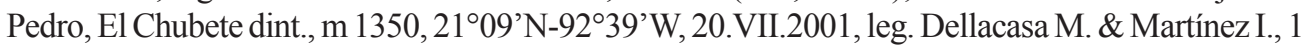

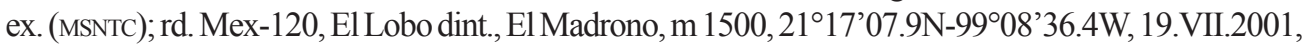
leg. Dellacasa M. \& Martínez I., 1 ex. (MSNTC); rd. Pinal de Amoles-Peña Blanca, La Quebrada, m 2300, $21^{\circ} 07^{\prime}$ N-99 $38^{\circ}$ 'W, 20.VII.2001, leg. Dellacasa M. \& Martínez I., 14 exx. (MSNTC, DCG); San Pedro Escalena, 4 Km ante, 21 ${ }^{\circ} 09^{\prime} 35.1^{\prime \prime N}-99^{\circ} 34^{\prime 2} 28.9^{\prime \prime} \mathrm{W}, \mathrm{m}$ 1784, 3.VII.2005, leg. Dellacasa M. \& Martínez I., 12 exx. (MSNTC); Xilitla, 20.5 rd mi W, 15.VII.1994, leg. Lago P. \& Zuccaro A. E., 2 exx. (DCG); Sinaloa: Concordia, 38 mi. NE, 6200 ft., 19.VI.1982, leg. Andrews F. G. (cowdung), 39 exx. (CSCAG); idem, 19.VI.1982, leg. Andrews F. G. (blacklight), 17 exx. (CSCAG); Concordia, 38 mi. NE, nr. Loberas, 3.VII.1982, leg. Andrews F. G. (cowdung), 5 exx. (CSCAG); idem, 3.VII.1982, leg. Andrews F. G. (blacklight), 1 ex. (CSCAG).

\section{CEPHALOCYCLUS ORDONEZI SP. NOV.}

(Figs. 40-43)

Type locality: México, Mpio. Chalco, Valle Espinoso.

Type repository: Museo de Zoología, FES Zaragoza, U.N.A.M., Iztapalapa, D.F. (Mexico).

Description: length 5.5-6.0 mm; shortly oval, moderately convex, subshiny, glabrous. Head and pronotum piceous; clypeal margin, pronotal sides, elytra, legs and antennal club reddishbrown. Head with epistoma feebly convex on disc, finely, sparsely, irregularly punctured; clypeus subtruncate anteriorly, widely rounded at sides, rather thickly bordered, the edge elongately ciliate laterally; genae acutely angulate, elongately ciliate, distinctly protruding more than eyes; frontal suture finely impressed; front very finely and sparsely punctured. Pronotum short, strongly transverse, feebly convex, faintly explanate laterally, subshiny, very finely, sparsely and subregularly punctured; sides almost parallel, distinctly bordered, the edge glabrous; hind angles obtusely rounded; base feebly bisinuate not bordered. Scutellum somewhat concave, finely punctured basally. Elytra oval, rather convex, finely striate; striae superficially punctured, not crenulate; interstices flat, sparsely and finely punctured; epipleural carina subdenticulate at shoulder. Hind tibiae superior apical spur as long as first tarsomere; latter as long as following three combined. Male: head and pronotum relatively larger and less convex; genae more strongly angulate; fore tibiae apical spur abruptly downward bent and outward curved; aedeagus fig. 42 . Female: head and pronotum relatively smaller and more convex; genae less angulate; fore tibiae apical spur slender and straight. 
Collecting notes: found in cattle dung.

Distribution: Mexico (Edo. de México, Puebla).

Derivatio nominis: named in honour of M. Ordóñez Reséndiz, curator in Museo de Zoología, FES Zaragoza, U.N.A.M., Iztapalapa, D.F. (Mexico).

Type material: Mexico - Edo. de México: Altzomoni, Mpio. Amecameca, m 3800, 1907’14.7"N98³9`34.5"W, 7.VI.2001, leg. Ordóñez \& Mora Y. (zacatonal alpino), 3 exx. (paratypes, unAm); La Tijera, Mpio. Amecameca, m 3100, 1904'10.6"N-9841'43.4"W, 8.VI.2001, leg. Mora Y. (bosque mixto), 10 exx. (paratypes, unAM, DCG); Paso de Cortés, Mpio. Amecameca, m 3700, 1905’07.8"N$98^{\circ} 38^{\prime} 45.2^{\prime \prime}$ W, 7.VI.2001, leg. Mora Y. (zacatonal alpino), 1 ex. (paratype, unAm); Rancho Los Venados, Mpio. Chalco, m 3100, 19¹7’37.1"N-98³9’18.7"W, 15.VI.2002, leg. Ordóñez M. \& Mora Y. (bosque pino), 21 exx. (paratypes, uNAM, DCG); Río Frío env., 19²0’37.7"N-98²0’28.8"W, m 2991, 8.VII.2005, leg. Dellacasa M., Martínez I., Cabrero F. \& Trotta N., 1 ex. (paratype, DCG);


(bosque de pino), 33 exx. (holotype male, allotype female and paratypes; UnAM, DCG); Puebla: carr. San Nicolas de los Ranchos-Paso de Cortes, $19^{\circ} 05^{\prime} 50.5^{\prime \prime} \mathrm{N}-98^{\circ} 36^{\prime} 51.0^{\prime \prime} \mathrm{W}, \mathrm{m} 3350,10 . \mathrm{VII} .2006$, leg. Dellacasa M., Fresi C. \& Martínez I., cattle dung, 57 exx. (paratypes, DCG, MSNTC).

\section{CEPHALOCYCLUS POTOSINUS SP. NOV.}

(Figs. 43-45)

Type locality: Cerro Potosí, 10300', Nuevo Léon, Mexico.

Type repository: Canadian National Collection. Ottawa.

Description: length 5.0-6.0 mm, oval-elongate, convex, moderately shiny, glabrous. Head and pronotum piceous, with clypeal margin and sides vaguely reddish respectively; elytra dark reddish-brown; legs brown-reddish; antennal club dark brownish. Head with epistoma feebly convex, flattened toward clypeal margin, finely microreticulate, very finely punctured on disc, punctation coarser distally; clypeus subtruncate anteriorly, widely rounded at sides, distinctly bordered, the edge upturned and laterally ciliate; genae obtuse, elongately ciliate, protruding more than eyes; front evenly, almost sparsely, distinctly punctured. Pronotum transverse, rather widely flattened at sides, superficially microreticulate, evenly finely sparsely punctured; sides feebly arcuate, lateral margin thickly bordered, the edge sparsely ciliate; hind angles obtusely rounded; base feebly bisinuate, not bordered. Scutellum flat, microreticulate, confusedly punctured. Elytra convex, oval-elongate, rather deeply striate; striae distinctly punctured, subcrenulate; interstices flat, finely microreticulate so few shiny, very finely and sparsely punctured; epipleural margin sparsely ciliate. Hind tibiae superior apical spur as long as first tarsomere; latter somewhat longer than following two combined. Male: fore tibiae apical spur relatively stouter and more abruptly downward bent; head and pronotum more transverse, less convex, more sparsely punctured; aedeagus fig. 45 . Female: fore tibiae apical spur relatively slender and less abruptly downward bent; head and pronotum narrowed frontwardly, more convex and more densely punctured.

Collecting notes: found in cattle and horse dung.

Distribution: Mexico (Coahuila, Nuevo León).

Etimology: the species is named after its type locality. 
Type material: Mexico - Coahuila: Paraíso Escondido, 25¹3'29.0"N-100²3'29.8"W, m 2476, 5.VII.2006, leg. Dellacasa M., Fresi C. \& Martínez I., 2 exx. (paratypes, DCG); Rancho San Francisco, $2^{\circ} 23^{\prime} 28.5^{\prime \prime N}-100^{\circ} 37^{\prime} 16.7^{\prime \prime} \mathrm{W}, \mathrm{m}$ 2220, 3.VII.2006, leg. Dellacasa M., Fresi C. \& Martínez I., horse dung, 22 exx. (paratypes, DCG, MSNTC); Nuevo León: Cerro Potosí, 10.300', 15-16.VII.1963, leg. Howden H., 6 exx. (paratypes, CNCO, DCG); idem, 15-16.VII.1963, leg. Howden H., 4 exx. (holotype male, allotype female, paratypes; HAнCO); Cerro El Potosí, $24^{\circ} 52^{\prime} 28.1^{\prime \prime N}-100^{\circ} 13^{\prime} 14.9^{\prime \prime} \mathrm{W}, \mathrm{m} 3274$, 4.VII.2006, leg. Dellacasa M., Fresi C. \& Martínez I.,

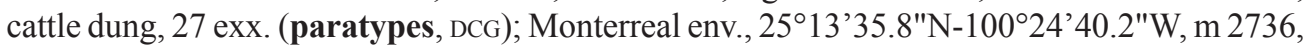
3.VII.2006, leg. Dellacasa M., Fresi C. \& Martínez I., horse dung, 6 exx. (paratypes, DCG).

\section{CEPHALOCYCLUS PULLATUS (SCHMIDT A., 1913)}

(Figs. 46-48)

Aphodius (Platyderus) pullatus; Schmidt A., 1913: 123.

Aphodius (Platydera) pullata; Schmidt A., 1913: 176.

Aphodius (Platyderides) pullatus; Schmidt A., 1922: 46; Dellacasa M., 1988: 252.

Cephalocyclus pullatus; Dellacasa M. \& alii, 2000: 14 (lectotype designation and new combination).

Type locality: Sierra de Durango [Mexico].

Type repository: Naturhistoriska Riksmuseet, Stockholm.

Redescription: length 4.5-5.0 mm; stout, oval, strongly convex. Head, pronotum and scutellum shiny, elytra strongly microreticulate thus dull, shortly pubescent. Dark reddish-brown; clypeal margin, pronotum sides and elytral apex vaguely reddish; legs reddish with darker tarsi; antennal club piceous. Head almost broad; epistoma nearly flat, superficially microreticulate, finely regularly and rather densely punctured; clypeus subtruncate anteriorly, widely rounded at sides, thinly but very distinctly bordered, edge sparsely ciliate; genae acutely angulate, rather elongately ciliate, strongly protruding more than eyes; frontal suture very finely impressed; front slightly more coarsely and more sparsely punctured than epistoma. Pronotum short and strongly transverse, moderately convex on disc, widely flattened at sides, somewhat depressed toward front and hind angles; rather finely, regularly and densely punctured; nearly parallel-sided; lateral margins distinctly bordered, edge glabrous; hind angles obtusely rounded; base not bordered, feebly bisinuate. Scutellum nearly flat, superficially microreticulate, sparsely punctured on basal half. Elytra shortly oval; rather convex; finely striate, striae shiny, rather deep, very superficially punctured, not crenulate; juxtasutural interstice moderately convex, rather superficially microreticulate thus somewhat shiny, finely but distinctly punctured; second and third interstices feebly convex from base till the half of disc, than flat and strongly microreticulate as all other interstices; latter strongly microreticulate, faintly punctured and with two lateral rows of very short yellowish hairs. Hind tibiae superior apical spur somewhat shorter than first tarsomere; latter as long as following three combined. Male: head and pronotum relatively larger and less convex; fore tibiae apical spur stout and abruptly downward bent; aedeagus fig. 48. Female: head and pronotum relatively narrower and more convex; fore tibiae apical spur slender and straight.

Collecting notes: none.

Distribution: Mexico (Durango, Sinaloa). 
Material examined: MexIco - Durango: El Salto, 20 Km W, 2.VIII.1998, leg. Caterino M. S., 2 exx. (EMEB); El Salto, 3 mi. W, 11.VII.1964, leg. Howden H. F., 1 ex. (USNMw); Res. Biosf. La Michilía, Piedra Herrada, 8.VIII.1994, leg. Anduaga S., 4 exx. (DCG); Sinaloa: Loberas (50 Km NE Concordia), 2.VIII.1998, leg. Caterino M. S., 16 exx. (EMEB).

\section{CEPHALOCYCLUS PUNCTICEPS (HAROLD, 1862)}

(Figs. 49-51)

Aphodius puncticeps Harold, 1862: 380; Bates, 1887: 91; Blackwelder, 1944: 213.

Aphodius (Platyderus) puncticeps; Schmidt A., 1913: 123.

Aphodius (Platyderides) puncticeps; Schmidt A., 1922: 47; Dellacasa M., 1988: 185.

Cephalocyclus puncticeps; Dellacasa M. \& alii, 1998: 149 (lectotype designation and new combination); Deloya \& Ibáñez-Bernal, 2000: 323; Dellacasa M. \& alii, 2002: 219.

Type locality: Chalchicomula [Ciudad Serdán], Puebla (Mexico).

Type repository: Muséum national d'Histoire naturelle. Paris.

Redescription: length 3.5-4.5 mm; oblong, moderately convex, very shiny. Head and pronotum blackish, latter obscurely reddish at sides; elytra piceous or obscurely testaceous, antennal club testaceous, legs piceous. Head with epistoma nearly flat, regularly and rather densely punctured particularly at sides; clypeus subtruncate, largely rounded and elongately bristled at sides, quite thinly bordered; genae acutely angulate, elongately bristled, protruding much more than eyes; frontal suture faint. Pronotum transverse, moderately convex, flattened at sides, irregularly rather sparsely punctured; lateral margins feebly rounded, distinctly bordered, sparsely elongately thinly bristled; hind angles rounded; base bisinuate, not bordered. Scutellum flat, superficially microreticulate and with some superficial punctures irregularly scattered. Elytra feebly widened posteriorly, strongly striate; striae distinctly punctured, crenulate; interstices distinctly convex on disc, nearly flat on preapical declivity, microreticulate, distinctly punctured, punctures almost regularly aligned along striae, shortly sparsely pubescent toward apex; epipleurae wide, elongately bristled on basal two thirds; shoulder not denticulate. Hind tibiae superior apical spur slightly longer than first tarsomere; latter shorter than following three combined. Claws stout, angulately broadened at base. Male: head relatively wider and less convex; pronotum not narrowed frontwardly; fore tibiae tibiae slender and elongate, apical spur hooked apically; aedeagus fig. 51. Female: head relatively narrower and more convex; pronotum narrowed frontwardly; fore tibiae stouter and rather short, apical spur regularly acuminate.

Collecting notes: none.

Distribution: Mexico (Jalisco, Edo. de México, Puebla).

\section{CEPHALOCYCLUS ROCKEFELLERI SP. NOV.} (Figs. 52-54, 66)

Type locality: Santa Barbara., Santa Barbara Distr., Chihuahua (Mexico).

Type repository: American Museum of Natural History. New York.

Description: length 4.5-6.0 mm; oblong, moderately convex, strongly shiny, glabrous. Reddishbrown, clypeal margin and pronotal sides paler; legs brownish with tarsi paler; antennal club dark 
brown. Head with epistoma almost flat, very finely sparsely punctured, punctation somewhat coarser distally; clypeus subtruncate anteriorly, widely rounded at sides, thinly bordered, the edge moderately upturned and elongately ciliate at sides; genae subangulate, posteriorly truncate, elongately and rather sparsely ciliate, distinctly protruding more than eyes; latter rather large. Pronotum transverse, moderately convex, doubly finely and rather sparsely punctured, large punctures two to three times larger than small ones; sides regularly arcuate, distinctly bordered, the edge sparsely elongately ciliate; hind angles widely rounded; base feebly bisinuate, not bordered. Scutellum flat, superficially sparsely punctured on basal half. Elytra elongate, rather convex, almost parallelsided; striae feebly impressed, superficially punctured, slightly crenulate; interstices feebly convex, sparsely irregularly and very finely punctured. Hind tibiae superior apical spur as long as first tarsomere; latter as long as following two combined. Male: head and pronotum relatively wider and less coarsely punctured; fore tibiae more elongate with apical spur stouter; middle tibiae inferior apical spur spatulate, outwardly curved (fig. 66); first mesotarsomere cylindrically plumped, inwardly deeply longitudinally grooved; aedeagus fig. 54 . Female: head and pronotum relatively narrower and more coarsely punctured; fore tibiae apical spur slender; middle tibiae inferior apical spur regularly acuminate; first mesotarsomere normally shaped.

Collecting notes: none.

Distribution: Mexico (Chihuahua).

Derivatio nominis: named in honour of David Rockefeller who funded a Mexican Expedition in 1947.

Type material: Mexico - Chihuahua: Cañon Prieto nr. Primavera, 6500-6800', 2.VII.1947, leg. Gertsch, D. Rockefeller Exp., 1 ex. (paratype, AMnHNY); Parral, 15 mi. E, 5500', 15.VII.1947, leg. Cazier, D. Rockefeller Exp., 1 ex. (paratype, AMNHNY); idem, 15.VII.1947, leg. Michener, D. Rockefeller Exp., 3 exx. (paratypes, AMNHNY); Primavera, 5500-6000', 30.VI.1947, leg. Cazier, D. Rockefeller Exp., 3 exx. (paratypes, AMNHNY); Santa Barbara, 63 mi. W, 5500', 20.VII.1947, leg. Cazier, D. Rockefeller Exp., 142 exx. (paratypes, AMNHNY); idem, 20.VII.1947, leg. Gertsch, D. Rockefeller Exp., 9 exx. (paratypes, AMNHNY); idem, 20.VII.1947, leg. Michener, D. Rockefeller Exp., 4 exx. (paratypes, AMNHNY); idem, 20.VII.1947, leg. Schramel, D. Rockefeller Exp., 6 exx. (paratypes, AMNHNY); Santa Barbara, Santa Barbara Dist., 6300’, 17.VII.1947, leg. Cazier, D. Rockefeller Exp., 86 exx. (holotype male, allotype female, paratypes; AMNHNY); idem, 17.VII.1947, leg. Michener, D. Rockefeller Exp., 41 exx. (paratypes, AMNHNY, DCG); idem, 17.VII.1947, leg. Schramel, D. Rockefeller Exp., 69 exx. (paratypes, AMNHNY, DCG); idem, 17.VII.1947, leg. Spieth, D. Rockefeller Exp., 6 exx. (paratypes, AMNHNY); Valle de Olivos, 5500', 20.VII.1947, leg. Cazier, D. Rockefeller Exp., 109 exx. (paratypes, AMNHNY, DCG); idem, 20.VII.1947, leg. Gertsch, D. Rockefeller Exp., 54 exx. (paratypes, AMNHNY); idem, 20.VII.1947, leg. Schramel, D. Rockefeller Exp., 4 exx. (paratypes, AMNHNY); idem, 20.VII.1947, leg. Spieth, D. Rockefeller Exp., 3 exx. (paratypes, AMNHNY).

\section{CEPHALOCYCLUS STEBNICKAE DELOYA\& IBÁÑEZ-BERNAL, 2000}

(Figs. 55-57)

Cephalocyclus stebnickae Deloya \& Ibáñez-Bernal, 2000: 320.

Type locality: Teopisca (11 miles N), Chiapas, Mexico.

Type repository: Instituto de Ecología, A. C. Xalapa, Veracruz, Mexico.

Redescription: length 7.0-8.5 mm, elongate, feebly convex, moderately shiny; almost glabrous, elytra on preapical declivity with extremely short, nearly imperceptible, yellowish hairs. Head 

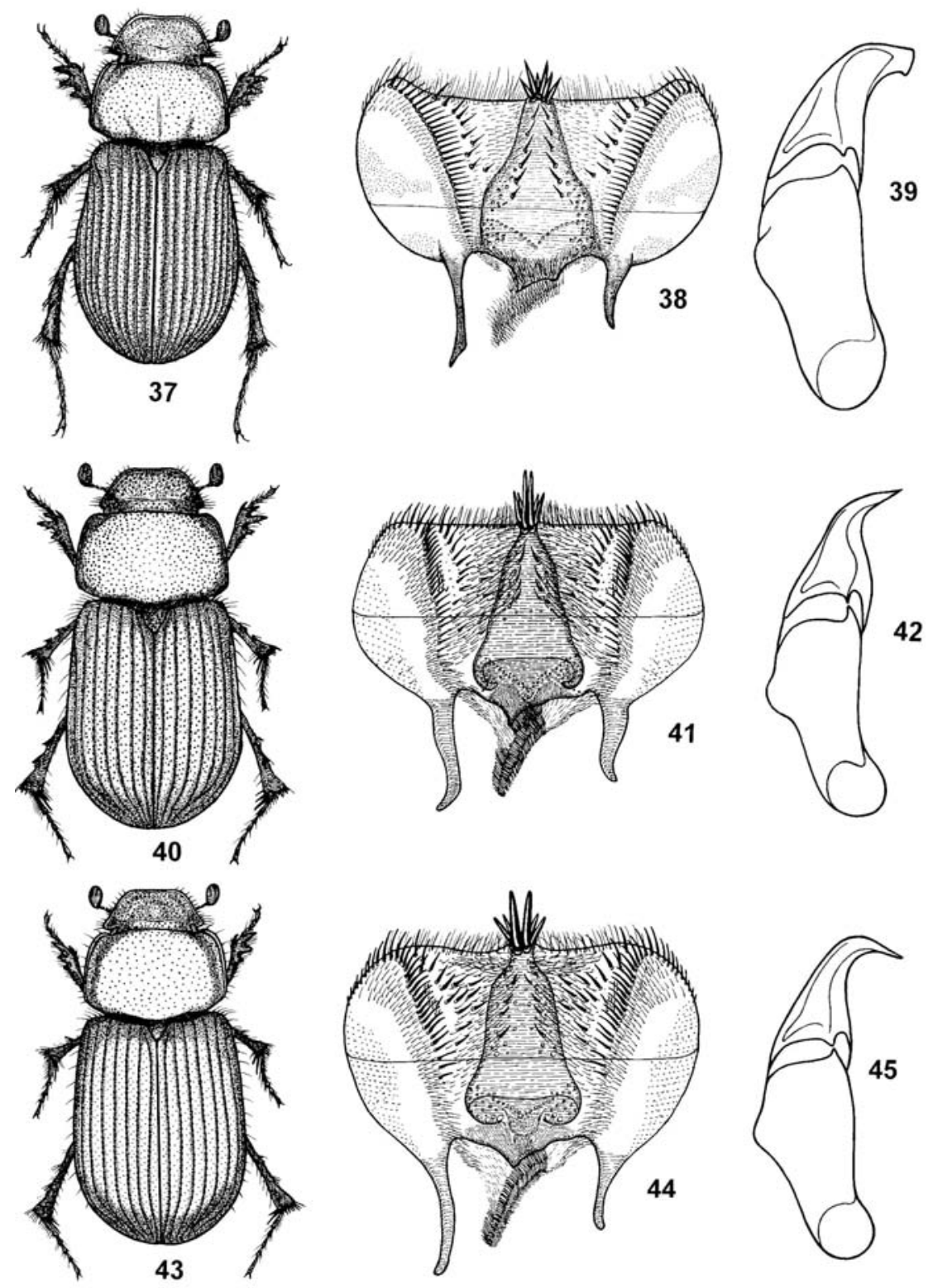

Figures 37-45

37-39.- Cephalocyclus mexicanus: 37.- habitus of male (length $6.0 \mathrm{~mm}$; Maravatío, Edo. Michoachán, Mexico); 38.- epipharynx; 39.- aedeagus (lateral view); 40-42.- Cephalocyclus ordonezi: 40.- habitus of male (length 5.5 mm; Valle Espinoso, Mpio. Chalco, Edo. México, Mexico); 41.- epipharynx; 42.- aedeagus (lateral view); 43-45.- Cephalocyclus potosinus: 43.- habitus of male (length $6.0 \mathrm{~mm}$; Cerro Potosí, Edo. Nuevo León, Mexico); 44.- epipharynx; 45.- aedeagus (lateral view). 
Dellacasa et al.: Revision of genus Cephalocyclus and ten new species
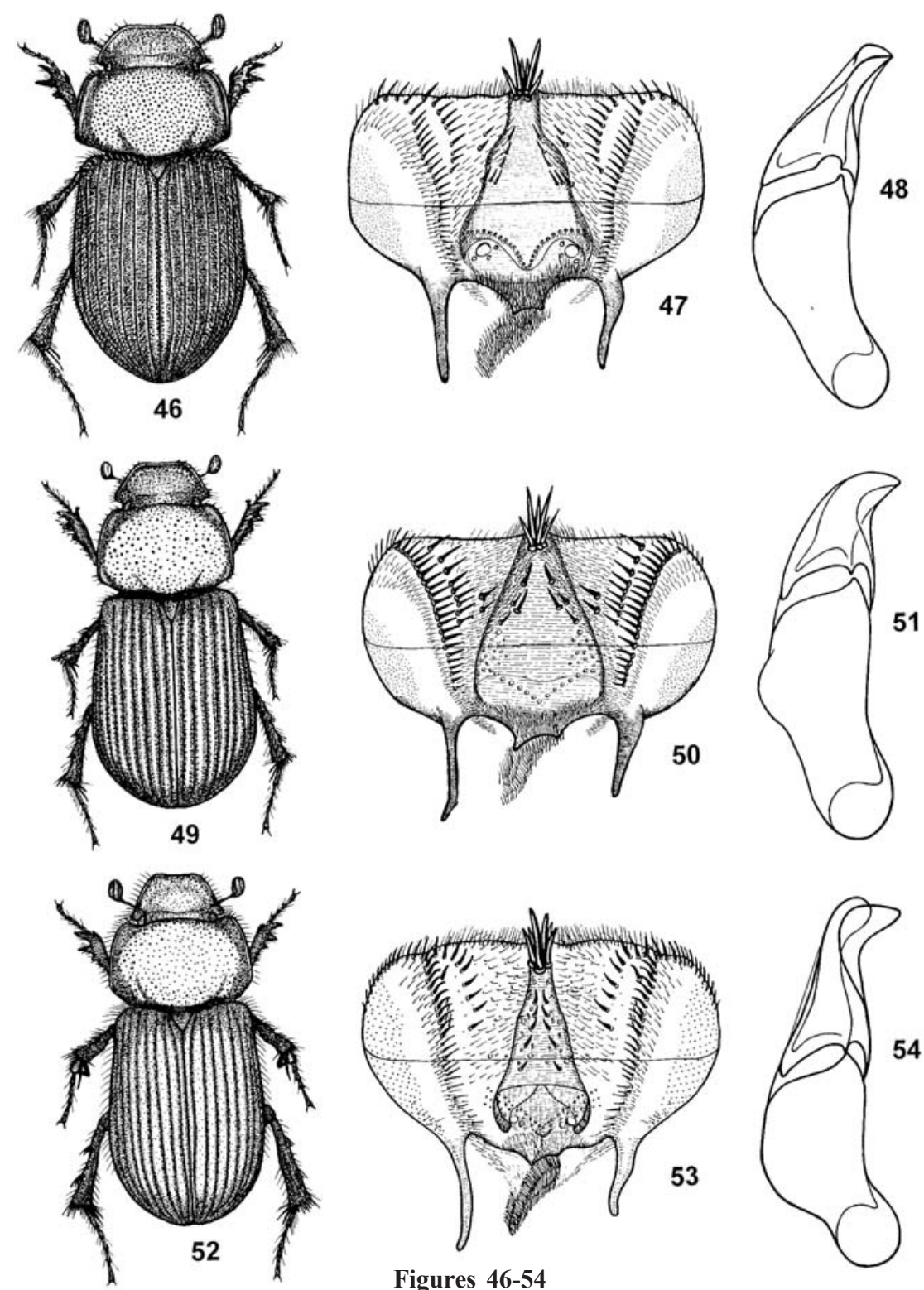

46-48.- Cephalocyclus pullatus: 46.- habitus of male (length $5.0 \mathrm{~mm}$; Piedra Herrada, Reserva de la Biosfera "La Michilía", Edo. Durango, Mexico); 47.- epipharynx; 48.- aedeagus (lateral view); 49-51.- Cephalocyclus puncticeps: 49.- habitus of male (length $4.0 \mathrm{~mm}$; Estación cientifica Los Soyos, Sierra de Manantlán, Edo. Jalisco, Mexico); 50.- epipharynx; 51.- aedeagus (lateral view); 52-54.- Cephalocyclus rockefelleri: 52.habitus of male (length $6.0 \mathrm{~mm}$; Santa Barbara, Santa Barbara Dist., Edo. Chihuahua, Mexico); 53.epipharynx; 54.- aedeagus (lateral view). 
and pronotum piceous; clypeal margin and pronotal sides vaguely brownish; elytra brownish with sutural margin darker; legs pale brown; antennal club testaceous. Head with epistoma almost flat, superficially microreticulate, finely, few densely and subregularly punctured, punctation somewhat denser and coarser distally; clypeus subtruncate anteriorly, widely rounded at sides, thickly bordered, edge glabrous and moderately upturned; genae angulate, elongately ciliate, strongly protruding more than eyes; frontal suture obsolete; front somewhat more finely and superficially punctured than epistoma. Pronotum transverse, feebly convex, narrowly flattened on sides, more widely flattened toward anterior angles, rather superficially and somewhat irregularly punctured, punctation finer anteriorly, denser and coarser toward base and on sides; latter very thickly bordered, edge glabrous; hind angles obliquely truncate and inwardly feebly sinuate, thinly bordered; base distinctly bisinuate, not bordered. Scutellum concave, faintly carinate at middle, with few sparse punctures on sides. Elytra oval-elongate, moderately convex, feebly widened barckwardly, epipleural margin thick, elongately ciliate at basal half; striae almost superficial, distinctly punctured, not crenulate; interstices superficially microreticulate, slightly convex, distinctly irregularly punctured, almost imperceptibly pubescent preapically. Hind tibiae superior apical spur as long as first tarsomere; latter somewhat shorter than following three combined. Male: head relatively larger; pronotum more strongly transverse; fore tibiae apical spur stout, subspatulate, abruptly downward bent; metasternal plate concave, distinctly microreticulate so almost dull, with few large punctures near lateral margin; aedeagus fig. 57. Female: head relatively smaller; pronotum narrowed frontwardly; fore tibiae apical spur slender, regularly acuminate from base to apex, feebly bent downward; metasternal plate slightly incavate, shiny, spersely and superficially punctured throughout.

Collecting notes: found in cattle and horse dung.

Distribution: Mexico (Chiapas).

Remarks: though Marco Dellacasa looked for types, they were not found in the collections of the repositories mentioned. The species is illustrated on topotypical specimens preserved in the Dellacasa collection.

Material examined: Mexico - Chiapas: El Chivero dint., San Cristóbal de las Casas, $2100 \mathrm{~m}$, $16^{\circ} 40^{\prime} 27.1^{\prime \prime N}-92^{\circ} 30^{\prime} 23.1 " \mathrm{~W}, 3 . V I I .2002$, leg. Dellacasa M. \& Martínez I., 2 exx. (MSNTC); Grutas Rancho Nuevo, San Cristóbal de las Casas, 2100 m, 1640'14.9"N-92³5'03.7"W, 3.VII.2002, leg. Dellacasa M. \& Martínez I., 53 exx. (MSNTC); idem, 16 40'14.9"N-92³5’03.7"W, m 2100, 18.V.2003, leg. Pacheco C., 10 exx. (MSNTC); idem, 29.VI.2003, leg. Pacheco C., 72 exx. (MSNTC, DCG); idem, 13.VII.2003, leg. Pacheco C., 8 exx. (MSNTC); San Cristóbal, 5 km E, 17.V.1990, leg. Howden H. \& A., 2 exx. (нансо); San Cristóbal, 10 Km E, 23.VI.1989, leg. Zuccaro E. \& Lago P., 10 exx. (DBUMu); San Cristóbal, 4 mi. E, 26.V.1969, leg. Bright D. E., 1 ex. (CNCO); San Cristóbal de las Casas, 6 mi. E, 7.V.1969, leg. Howden H., 9 exx. (нанСо); San Cristóbal, 7 mi. E, 30.V.1969, leg. Bright D. E., 16 exx. (CNCO); San Cristóbal de las Casas, 8 mi. NE, 5.V.1969, leg. Howden H., 132 exx. (нансо); San Cristóbal de las Casas, 3 mi. NW, 29.V.1969, leg. Howden H., 2 exx. (HАнCO); San Cristóbal, 15 km SE, 11.VI.1989, leg. Howden H., 12 exx. (HAнCо); idem, m 2600, 26.V.1990, leg. Howden H. \& A., 8 exx. (CNCO, HAHCO); San Cristóbal, 8 mi. SE, 17.V.1969, leg. Campbell J. M., 13 exx. (CNCO); San Cristóbal de las Casas, 5 km W, 8000', 13-16.VII.1969, leg. Peck S. \& J. (pine-oak forest), 4 exx. (нанСо); San Cristóbal, Hwy 199, 11 Km NE, 8000 ft., 25.V.1987, leg. Rider D. A., E. G. \& Riley T. J. (mercury vapor \& blacklight), 13 exx. (LSAMBR, DCG); idem, 28.V.1987, leg. Rider D. A., E. G. \& Riley 
Dellacasa et al.: Revision of genus Cephalocyclus and ten new species
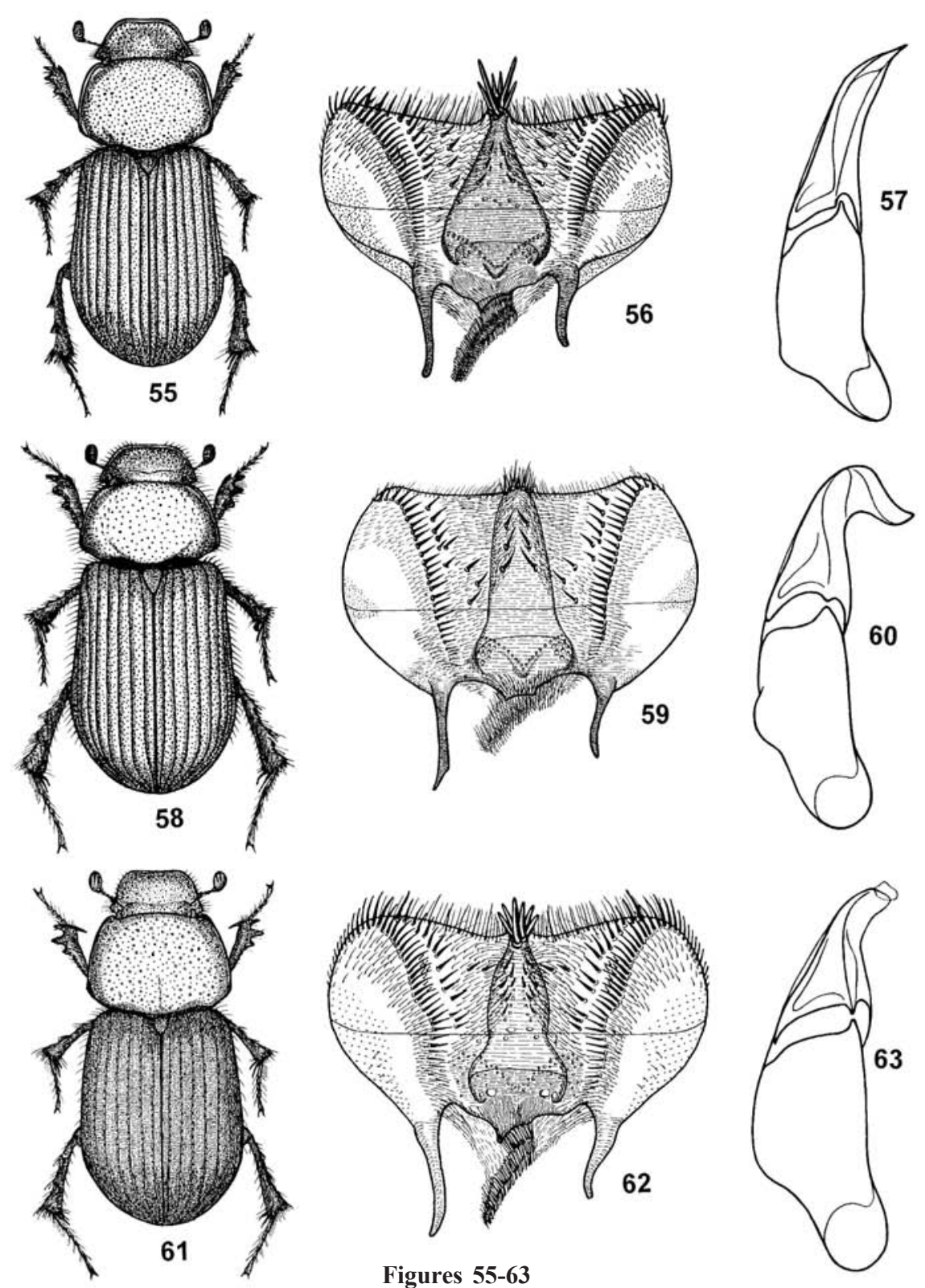

55-57.- Cephalocyclus stebnickae: 55.- habitus of male (length $8.0 \mathrm{~mm}$; Grutas Rancho Nuevo, San Cristóbal de las Casas, Edo. Chiapas, Mexico); 56.- epipharynx; 57.- aedeagus (lateral view); 58-60.- Cephalocyclus villosipes: 58.- habitus of male (length $9.0 \mathrm{~mm}$; Zacualtipán, Edo. Hidalgo, Mexico); 61-63.- Cephalocyclus carmenae: 61.- habitus of male (length $6.5 \mathrm{~mm}$; Monterreal env., $25^{\circ} 13^{\prime} 35.8^{\prime \prime} \mathrm{N}-100^{\circ} 24^{\prime} 40.2^{\prime \prime} \mathrm{W}$, Edo. Coahuila, Mexico); 62.- epipharynx; 63.- aedeagus (lateral view). 
T. J. (mercury vapor \& blacklight), 3 exx. (LSAMBR); Mt. Tzontehuitz, 9500', 17.V.1969, leg. Campbell J. M., 16 exx. (CNCO); Teopisca, 17 km N, 13.VI.1989, leg. H. Howden, 11 exx. (HAHCO); Teopisca, 10 mi. E, 11-12.V.1969, leg. Howden H., 1 ех. (нанСо); idem, 2.VI.1969, leg. Howden H., 9 ехx. (нанСо).

\section{CEPHALOCYCLUS VILLOSIPES (HAROLD, 1862)}

(Figs. 58-60, 68)

Aphodius villosipes Harold, 1862: 384; Bates, 1887: 92; Blackwelder, 1944: 213.

Aphodius (Platyderus) villosipes; Schmidt A., 1913: 123.

Aphodius (Platyderides) villosipes; Schmidt A., 1922: 50; Dellacasa M., 1988: 214.

Cephalocyclus villosipes; Dellacasa M. \& alii, 1998: 151 (lectotype designation and new combination); Deloya \& Ibáñez-Bernal, 2000: 322; Dellacasa M. \& alii, 2002: 220.

Type locality: Mexico.

Type repository: Muséum national d'Histoire naturelle. Paris.

Redescription: length 8.0-9.0 mm; subdepressed, elongate, moderately shiny. Piceous-reddish, antennal club and legs paler. Head with epistoma feebly gibbous, superficially microreticulate, subrugosely punctured toward clypeal margin, superficially sparsely rather irregularly punctured at middle; clypeus anteriorly subtruncate, widely rounded and elongately bristled at sides, quite strongly bordered, edge upturned; genae acute, elongately densely bristled, protruding much more than eyes; frontal suture finely impressed. Pronotum feebly convex, moderately transverse, weakly flattened on sides, slightly densely irregularly punctured, at sides punctures provided with elongate setae curved posteriorly; lateral margins feebly rounded, strongly bordered, bristled; hind angles widely rounded; base lobate at middle and distinctly bisinuate. Scutellum faintly depressed at middle, microreticulate, coarsely irregularly punctured. Elytra elongate, feebly widened in apical half, distinctly striate; striae superficially punctured, not crenulate; interstices feebly convex, microreticulate, distinctly irregularly sparsely punctured, very strongly microreticulate toward apex so rather dull, very confusedly and relatively less coarsely and densely punctured; epipleurae rather narrow, elongately sparsely bristled on basal two thirds; shoulder not denticulate. Male: inferior apical spur of middle tibiae spatulate, outwardly curved (fig. 65); hind tibiae superior apical spur longer than first tarsomere; latter distinctly shorter than following three combined; aedeagus fig. 60. Female: inferior apical spur of middle tibiae regularly acuminate; hind tibiae superior apical spur as long as first tarsomere; latter as long as following three combined.

Collecting notes: none.

Distribution: Mexico (Hidalgo, Edo. de México, Oaxaca, Puebla, Veracruz).

Material examined: Mexico - Edo. de México: Real de Arriba, Temescaltepec, VII.1932, leg. Hinton H. E. \& Usinger R. L., 1 ex. (USNMw).

\section{AKNOWLEDGEMENTS}

Thanks are due to N. Adams (Washington), C. B. Barr (Berkeley, CA), C. Bellamy (Sacramento, CA), F. Cabrero-Sañudo (Xalapa, Veracruz), A. Davies (Ottawa), L. Herman (New York, NY), H. Howden (Ottawa), J. Jelínek (Prague), P. K. Lago (University, MS), J. McNamara (Ottawa), M. A. 
Moron (Xalapa, Veracruz), V. Moseley Bayless (Baton Rouge, LA), P. Perkins (Cambridge, MA), P. E. Skelley (Gainesville, FL) for loan of type material and support.
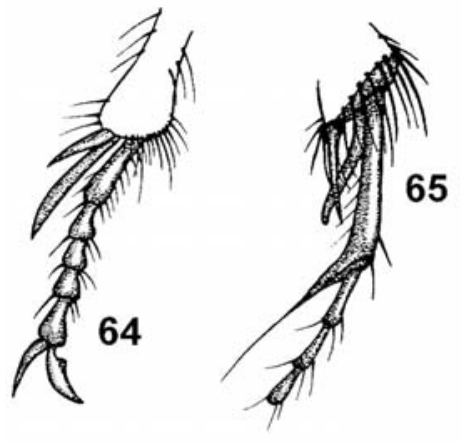
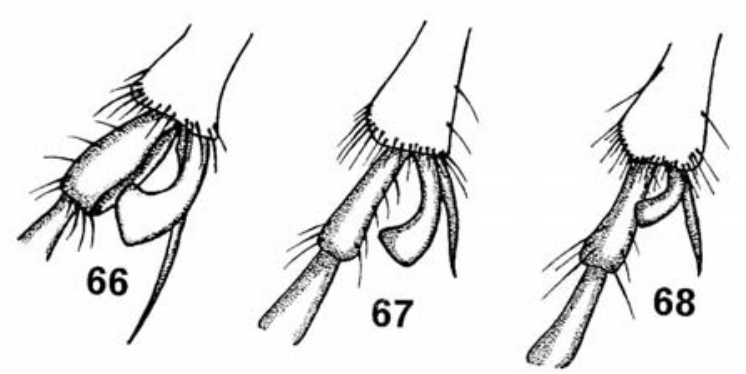

Figures 64-68

64.- Cephalocyclus fuliginosus, apex of right mesotibia and tarsus of male (ventral view); 65.- Cephalocyclus mexicanus, tarsomeres I-IV of right mesotarsus of male (ventral view); 66.- Cephalocyclus rockefelleri, apex of right mesotibia of male (ventral view); 67.- Cephalocyclus luridiventris, idem; 66.- Cephalocyclus villosipes, idem.

\section{LITERATURECITED}

Balthasar V. 1946. De novis generis Aphodius Illig. speciebus. Sbor. ent. Odd. nar. Mus. Praha, 24: 53-68.

Bates H. W. 1887. Biologia Centrali-Americana. Insecta. Coleoptera: Lamellicornia (Copridae, Aphodiidae, Orphnidae, Hybosoridae, Geotrupidae, Trogidae, Aclopidae, Chasmatopteridae, Molonthidae). 2: 25-160. 2:337-416.

1889. Biologia Centrali-Americana. Insecta. Coleoptera: Lamellicornia. (Suppl.).

Blackwelder R. E. 1944. Checklist of the Coleopterous insects of Mexico, Central America, the West Indies and South America. Part. II. Bull. U. S. nat. Mus., 185: 189-341.

Dellacasa G., P. Bordat \& M. Dellacasa, 2001. A revisional essay of world genus-group taxa of Aphodiinae. Mem. Soc. ent. ital., 79 [2000]: 1-482.

Dellacasa M. 1988. Contribution to a world-wide Catalogue of Aegialiidae, Aphodiidae, Aulonocnemidae, Termitotrogidae. (Part I). Mem. Soc. ent. ital. 66 [1987]: 1-455.

Dellacasa M., R. D. Gordon \& G. Dellacasa, 1998. The types of Aphodius species described by Harold from Mexico with description of a new genus. Acta Zool. Mex. (n. s.) 74: 130-162.

.2000. Aphodius (Platyderides) pullatus A. Schmidt, 1913, another Mexican taxon to be moved into the genus Cephalocyclus and remarks on Cephalocyclus mexicanus (Harold, 1862) and Aphodius freyi Balthasar, 1941. Acta. Zool. Mex. (n. s.) 79: 11-15.

. 2002. Aphodiinae described or recorded by Bates in Biologia Centrali-americana. Acta Zool. Mex. (n. s.) 86: 155-223.

Deloya C. 1998. Primer registro de Cephalocyclus mexicanus (Harold, 1862, para el Estado de Morelos, México. Folia entomol. Mex. 103: 101-102. 
Deloya C. \& S. Ibáñez-Bernal 2000. New species of Aphodiinae from Mexico and a key to species of Cephalocyclus Dellacasa, Gordon and Dellacasa. Col. Bul. 54 (3): 318-324.

Harold E. 1862. Beitrage zur Kenntniss einiger coprophagen Lamellicornien. (Viertes Stück). Berl. ent. Zeits. 6: 379-403.

.1863. Beitrage zur Kenntniss einiger coprophagen Lamellicornien. (Fünftes Stück). Berl. ent. Zeits. 7: 327-389.

.1871. Beitrage zur Kenntniss einiger coprophagen Lamellicornien. (Siebentes Stück). Berl. ent. Zeits. 15: 249-287.

Horn G. H. 1887. A monograph of the Aphodiini inhabiting the United States. Trans. Am. ent. Soc. 14: $1-110$.

Leng C. V. 1920. Catalogue of the Coleoptera of America, North of Mexico. Mount Vernon, N.Y., $1-470$.

Robinson M. 1940. Studies in the Scarabaeidae. II. Trans. Amer. ent. Soc., 66: 141-159.

Schmidt A. 1913. Erster Versuch einer Einteilung der exotischen Aphodien in Subgenera und als Anhang einige Neubeschreibungen. Arch. Naturg. Abt. A 79: 117-178.

. 1916. Namenänderungen und Beschreibung neuer Aphodiinen. Arch. Naturg. Abt. A 82: 95-116.

. 1922. Coleoptera Aphodiinae (Das Tierreich), Berlin \& Leipzig, 45: 1-614.

Recibido: 2 de febrero 2007

Aceptado: 13 de abril 2007 
Dellacasa et al.: Revision of genus Cephalocyclus and ten new species

\section{CATALOGUE}

Genus Cephalocyclus Dellacasa M. Gordon \& Dellacasa G. 1998 (Southern USA, Mexico, Guatemala, Costa Rica)

Type species: Aphodius fuliginosus Harold, 1863 (original designation)

\begin{tabular}{|c|c|c|}
\hline 1. & bordati sp. nov. & Mexico (Chihuahua, Durango) \\
\hline 2. & carmenae sp. nov. & Mexico (Coahuila, Nuevo León) \\
\hline 3. & cartagoensis sp. nov. & Costa Rica (Cartago) \\
\hline 4. & costaricensis sp. nov. & Costa Rica (Alejuela, Cartago, San José) \\
\hline \multirow[t]{2}{*}{5.} & durangoensis (Bates, 1887) & $\begin{array}{l}\text { Mexico (Coahuila, Durango, Michoacán, Nuevo } \\
\text { León, Oaxaca) }\end{array}$ \\
\hline & pugil (Balthasar, 1946) & \\
\hline 6. & fuliginosus (Harold, 1863) & $\begin{array}{l}\text { Guatemala, Mexico (Chihuahua, Distrito } \\
\text { Federal, Durango, Guanajuato, Hidalgo, Jalisco } \\
\text { Edo. de México, Michoacán, Morelos, Nayarit } \\
\text { Oaxaca, Puebla, Sinaloa, Tlaxcala, Zacatecas) } \\
\end{array}$ \\
\hline \multirow[t]{2}{*}{7.} & gravidus (Harold, 1863) & $\begin{array}{l}\text { Mexico (Chihuahua, Distrito Federal, Durango } \\
\text { Hidalgo, Jalisco, Edo. de México, Michoacán) }\end{array}$ \\
\hline & pinguescens (Harold, 1871) & \\
\hline 8. & halffteri sp. nov. & Mexico (Guerrero, Edo. de México) \\
\hline \multirow[t]{2}{*}{9.} & hogei (Bates, 1887) & $\begin{array}{l}\text { Guatemala, Mexico (Coahuila, Distrito Federal } \\
\text { Durango, Hidalgo, Edo. de México, Michoacán } \\
\text { Morelos, Oaxaca, Puebla, Querétaro, Veracruz) } \\
\text { U.S.A (Arizona)? }\end{array}$ \\
\hline & transversus (Robinson, 1940) & \\
\hline 10. & howdenorum sp. nov. & Mexico (Nuevo León) \\
\hline 11. & lagoi sp. nov. & $\begin{array}{l}\text { Mexico (Coahuila, Nuevo León, Querétaro, San } \\
\text { Luis Potosí) }\end{array}$ \\
\hline 12. & luridiventris (Harold, 1863) & Mexico (Distrito Federal, Oaxaca, Puebla) \\
\hline 13. & luteolus (Horn, 1887) & U.S.A. (Arizona, New Mexico, Texas, Utah) \\
\hline \multirow[t]{2}{*}{14.} & mexicanus (Harold, 1862) & $\begin{array}{l}\text { Guatemala, Mexico (Distrito Federal, Durango } \\
\text { Guerrero, Hidalgo, Jalisco, Edo. de México } \\
\text { Michoacán, Morelos, Nayarit, Oaxaca, Puebla } \\
\text { Querétaro, Sinaloa) }\end{array}$ \\
\hline & omiltemius (Bates, 1889) & \\
\hline 15. & ordonezi isp. nov. & Mexico (Edo. de México, Puebla) \\
\hline 16. & potosinus sp. nov. & Mexico (Coahuila, Nuevo León) \\
\hline 17. & pullatus (Schmidt A., 1913) & Mexico (Durango, Sinaloa) \\
\hline 18. & puncticeps (Harold, 1862) & Mexico (Jalisco, Edo. de México, Puebla) \\
\hline 19. & rockefelleri sp. nov. & Mexico (Chihuahua) \\
\hline 20. & stebnickae Deloya \& Ibáñez-Bernal, 2000 & Mexico (Chiapas) \\
\hline 21. & villosipes (Harold, 1862) & $\begin{array}{l}\text { Mexico (Hidalgo, Edo. de México, Oaxaca, } \\
\text { Puebla, Veracruz) }\end{array}$ \\
\hline
\end{tabular}

